\title{
Synaptic Targeting of Double-Projecting Ventral CA1 Hippocampal Neurons to the Medial Prefrontal Cortex and Basal Amygdala
}

\author{
W.Woong Bin Kim and Jun-Hyeong Cho \\ Department of Cell Biology and Neuroscience, University of California, Riverside, California 92521
}

The acquisition and retrieval of contextual fear memory requires coordinated neural activity in the hippocampus, medial prefrontal cortex (mPFC), and amygdala. The contextual information encoded in the hippocampus is conveyed to the mPFC and amygdala for contextual fear conditioning. Previous studies have suggested that a CA1 neuronal population in the ventral hippocampus ( $\mathrm{VH}$ ) projects to both the mPFC and amygdala and is recruited in context-dependent control of conditioned fear. However, how double-projecting ventral CA1 hippocampal (vCA1) neurons modulate the activity of the mPFC and amygdala at the synaptic level has not been determined previously. Here, we show that the optogenetic silencing of the $\mathrm{VH}$ prevented the recall of contextual fear memory in mice, indicating its role in contextual fear expression. In dual retrograde viral tracing and c-Fos immunostaining experiments, we found that a proportion of vCA1 neurons projected to both the $\mathrm{mPFC}$ and amygdala and were recruited preferentially during context exposure, suggesting their role in encoding context representations. Moreover, optogenetic stimulation of axon collaterals of double-projecting vCA1 neurons induced monosynaptic excitatory responses in both the MPFC and basal amygdala, indicating that they could convey contextual information through the VH-mPFC and VH-amygdala pathways. The activation of double-projecting vCA1 neurons also induced action potential firings in the mPFC neurons that project to the amygdala, suggesting that they can also activate the VH-mPFC-amygdala pathway. With these synaptic mechanisms, double-projecting vCA1 neurons could induce synchronized neural activity in the mPFC and amygdala and convey contextual information efficiently to the basal amygdala for contextual fear conditioning.

Key words: amygdala; fear conditioning; hippocampus; learning and memory; mPFC

Significance Statement

This work demonstrates that ventral CA1 hippocampal (vCA1) neurons projecting to both the medial prefrontal cortex (mPFC) and amygdala are activated preferentially when contextual information is processed in the ventral hippocampus, which is required for contextual fear expression. Our electrophysiological experiments reveal that the activation of double-projecting vCA1 neurons induces excitatory synaptic activity in both the mPFC and amygdala. These results suggest that double-projecting vCA1 neurons could contribute to contextual fear responses by inducing synchronized activity in the mPFC and amygdala and conveying contextual information to the basal amygdala more efficiently than vCA1 neurons projecting to either the mPFC or amygdala alone. These findings provide important insights into the mechanisms of the acquisition and retrieval of contextual fear memory.

\section{Introduction}

In contextual fear conditioning, experimental subjects learn to associate an environmental context with an aversive uncondi-

Received Nov. 18, 2016; revised March 29, 2017; accepted April 2, 2017.

Author contributions: J.-H.C. designed research; W.B.K. and J.-H.C. performed research; W.B.K. and J.-H.C. analyzed data; J.-H.C. wrote the paper.

ACKNOWLEDGEMENTS: We thank Dr. Rachael Neve at MIT for HSV packaging and Dr. Eric Kremer at CNRS for CAV2 packaging. This study was supported by Initial Complementary Funds from the University of California, Riverside (J.-H.C.).

The authors declare no competing financial interests.

Correspondence should be addressed to Jun-Hyeong Cho, Department of Cell Biology and Neuroscience, University of California, 900 University Avenue, Riverside, CA 92521. E-mail: juncho@ucr.edu. tioned stimulus (US; e.g., an electric foot shock), resulting in conditioned fear responses (e.g., freezing behavior) to the context (Maren et al., 2013). The acquisition of context-US associative memory requires two processes in the brain. Contextual representations are first encoded in the hippocampus (context encoding) and then associated with the US (context conditioning), which requires coordinated neural activity in the hippocampus, medial prefrontal cortex (mPFC), and amygdala (Helmstetter and Bellgowan, 1994; Muller et al., 1997; Corcoran and Quirk, 
2007; Goshen et al., 2011; Zhu et al., 2014). Therefore, hippocampal representations of a context are conveyed to the mPFC and amygdala for the context-US association. However, the synaptic mechanisms determining how contextual information is relayed to these areas for contextual fear conditioning have not been investigated thoroughly.

The hippocampus is characterized by multiple anatomical and functional domains along its longitudinal axis (Strange et al., 2014). The ventral hippocampus ( $\mathrm{VH})$ projects directly to the mPFC and amygdala (Jay and Witter, 1991; Kishi et al., 2006), whereas the dorsal hippocampus (DH) is connected to these areas indirectly through the entorhinal and perirhinal cortices (Pitkänen et al., 2000; Tanaka et al., 2014). The role of the DH neurons in context encoding and contextual fear expression has been well documented (Goshen et al., 2011; Liu et al., 2012; Denny et al., 2014). Although it encodes contextual representations with larger place fields and lower spatial selectivity than the DH (Jung et al., 1994; Kjelstrup et al., 2008), the VH also plays an essential role in contextual fear responses by conveying contextual information directly to the mPFC and amygdala. Previous studies with electrolytic lesions and pharmacological and chemogenetic inhibition have suggested that the $\mathrm{VH}$ plays a role in contextual fear conditioning (Maren and Fanselow, 1995; Bast et al., 2001; Zhang et al., 2001; Trivedi and Coover, 2004; Rudy and Matus-Amat, 2005; Pentkowski et al., 2006; Zhu et al., 2014; Zhang et al., 2015). Depending on their projection targets, ventral CA1 hippocampal (vCA1) neurons play different roles in various behavioral tasks, including anxiety-related behavior, goaldirected behaviors, and social discrimination (Ciocchi et al., 2015; Okuyama et al., 2016). A recent study by Xu et al. (2016) also implicates the pathway from the $\mathrm{VH}$ to the basal amygdala in the retrieval of contextual fear memory. Moreover, a population of vCA1 neurons project to both the MPFC and amygdala with axon collaterals and are recruited preferentially in contextdependent fear renewal (Ishikawa and Nakamura, 2006; Jin and Maren, 2015). It has been suggested that these double-projecting vCA1 neurons may facilitate synchronized activity in the mPFC and amygdala, which is implicated in the expression of conditioned fear (Lesting et al., 2011). However, it remains to be determined how these double-projecting vCA1 neurons modulate neural activity in the mPFC and amygdala for conditioned fear at the synaptic level.

Using a combined approach of viral tracing, optogenetics, c-Fos immunohistochemistry, and electrophysiology, we found that a significant proportion of vCA1 neurons projected to both the mPFC and amygdala with functional excitatory synapses and were recruited preferentially during context exposure in mice. Moreover, the activation of these vCA1 neurons induced excitatory postsynaptic responses in the mPFC and basal amygdala and action potential firings in $\mathrm{mPFC}$ neurons projecting to the basal amygdala. With these synaptic mechanisms, double-projecting vCA1 neurons encoding context representations could induce synchronized neural activity in the mPFC and amygdala and activate the basal amygdala more efficiently for the acquisition and retrieval of contextual fear memory.

\section{Materials and Methods}

Subjects. Six- to 8-week-old C57BL/6J mice of either sex (Jackson Laboratory, stock \#000664) underwent stereotaxic brain surgery. After surgery, the mice were housed singly in home cages on a $12 \mathrm{~h}$ light/dark cycle with food and water available continuously. All of the animal procedures were approved by the Institutional Animal Care and Use Committee of the University of California, Riverside.
Virus constructs. The recombinant adeno-associated viruses (AAV, serotype 5) were packaged by the Vector Core at the University of North Carolina. The AAV titer was $8.5 \times 10^{12}$ genome copies $(\mathrm{GCs}) / \mathrm{ml}$ for AAV-CaMKII-channelrhodopsin-2 (ChR2) (H143R)-eYFP, $6.6 \times 10^{12}$ $\mathrm{GCs} / \mathrm{ml}$ for AAV-double-floxed inverted open reading frame (DIO)ChR2(H143R)-eYFP, $4.0 \times 10^{12}$ GCs/ml for AAV-CaMKII-eArch3eYFP, and $4.3 \times 10^{12} \mathrm{GCs} / \mathrm{ml}$ for AAV-CaMKII-eYFP. Herpes simplex viruses (HSV) for the retrograde tracing experiments (HSV-pEF1 $\alpha$ eYFP and HSV-pEF1 $\alpha$-mCherry) were packaged by Dr. Rachael Neve at the Viral Gene Transfer Core Facility of the Massachusetts Institute of Technology and the titer was $>3.5 \times 10^{9}$ infectious units $/ \mathrm{ml}$. Canine adenovirus (CAV2-pCMV-Cre) was obtained from Dr. Eric Kremer at the Institute of Molecular Genetics of Montpellier and the titer was $5.15 \times 10^{12}$ viral particles $/ \mathrm{ml}$.

Stereotaxic virus injection and optical cannula implantation. Six- to 8 -week-old mice were used for stereotaxic surgery. Before surgery, general anesthesia was induced by placing the mice in a transparent anesthetic chamber filled with $5 \%$ isoflurane. The anesthesia was maintained during surgery with $1 \%$ isoflurane applied to the nostrils of the mice using a precision vaporizer. Mice were checked for the absence of the tail-pinch reflex as a sign of sufficient anesthesia. The mice were then immobilized in a stereotaxic frame with nonrupture ear bars (David Kopf Instruments) and ophthalmic ointment was applied to prevent eye drying. After making an incision to the midline of the scalp, small unilateral or bilateral craniotomies were performed using a microdrill with $0.5 \mathrm{~mm}$ burrs. The tips of glass capillaries loaded with AAV, HSV, or CAV2 were placed into specific brain areas using the following stereotaxic coordinates: $\mathrm{mPFC}: 1.7 \mathrm{~mm}$ rostral to bregma, $0.4 \mathrm{~mm}$ lateral to midline and $2.5 \mathrm{~mm}$ ventral to bregma; basal amygdala (the basolateral and basomedial nuclei of the amygdala): $1.6 \mathrm{~mm}$ caudal to bregma, 3.5 $\mathrm{mm}$ lateral to midline, and $4.8 \mathrm{~mm}$ ventral to bregma; and the CA1 area of the VH: $3.4 \mathrm{~mm}$ caudal to bregma, $3.7 \mathrm{~mm}$ lateral to midline, and 4.0 $\mathrm{mm}$ ventral to bregma (Franklin and Paxinos, 2008). A total of 0.15-1.0 $\mu \mathrm{l}$ of virus-containing solution was injected at a rate of $0.1 \mu \mathrm{l} / \mathrm{min}$ using a $10 \mu \mathrm{l}$ Hamilton microsyringe and a syringe pump. More specifically, $0.15 \mu$ l of AAV-pCaMKII-eYFP or AAV-pCaMKII-ChR2(H143R)-eYFP was injected into the $\mathrm{CA} 1$ area of the $\mathrm{DH}$ or $\mathrm{VH}$ (see Fig. 1); $1.0 \mu \mathrm{l}$ of AAV-pCaMKII-eArch3-eYFP or AAV-pCaMKII-eYFP into the vCA1 (see Fig. 2); $1.0 \mu \mathrm{l}$ of HSV-pEF1 $\alpha$-eYFP and $1.0 \mu \mathrm{l}$ of HSV-pEF1 $\alpha$ mCherry into the mPFC and basal amygdala, respectively (see Figs. 3, 4); $1.0 \mu \mathrm{l}$ of AAV-DIO-ChR2(H143R)-eYFP into the CA1 area of the VH; $1.0 \mu \mathrm{l}$ of CAV2-pCMV-Cre into the mPFC or basal amygdala (see Figs. 5, 6); and a mixture of $0.5 \mu \mathrm{l}$ of HSV-pEF1 $\alpha$-mCherry and $0.5 \mu \mathrm{l}$ of CAV2pCMV-Cre into the basal amygdala (see Fig. 7). After injection, the capillary was left in place for an additional $5 \mathrm{~min}$ to allow for diffusion of the virus solution and then withdrawn. For the optogenetic inhibition experiment (see Fig. 2), bilateral optical cannulae (200 $\mu \mathrm{m}$ in diameter, numeric aperture 0.53; Doric Lenses) were implanted into the CA1 area of the $\mathrm{VH}(3.4 \mathrm{~mm}$ caudal to bregma, $3.7 \mathrm{~mm}$ lateral to midline, and $3.5 \mathrm{~mm}$ ventral to bregma) and secured with dental cement. The scalp incision was closed with surgical suturing. After surgery, mice were given saline (1 $\mathrm{ml}$, s.c.) containing buprenorphine $(0.13 \mathrm{mg} / \mathrm{kg}$ body weight $)$ for analgesia and hydration. Behavioral training began 3 weeks after the viral injection surgery and electrophysiological recording experiments were performed 4 weeks after surgery.

Contextual fear conditioning and in vivo optogenetic inhibition. For the optogenetic inhibition experiment (Fig. $2 F$ ), mice were habituated with optical cannulae connected to the optical patch cable without laser stimulation for $10 \mathrm{~min}$ once per day for $6 \mathrm{~d}$ (day 1 to day 6) in context $\mathrm{B}$ (acrylic plate floor, no illumination, acetic acid odor). On the training day (day 7), mice were placed in context A (stainless steel grid floor, white light illumination, benzaldehyde odor) in a standard fear-conditioning chamber (Med Associates) without optical cannula connection. The fear-conditioning chamber was illuminated with a white house light when mice were placed in the context. Mice were given US $(0.5 \mathrm{~mA}$ electric foot shock for $2 \mathrm{~s}$ with $2 \mathrm{~min}$ intervals between the shocks) and received the first US $3 \mathrm{~min}$ after they were placed in the context. On the test days (days 8-10), mice were placed in context A with optical cannulae connected to the optical patch cable and a green laser. After a $1 \mathrm{~min}$ 
acclimatization period, mice were tested for freezing behavior in context A for $6 \mathrm{~min}$. After the baseline measurement of freezing behavior for 2 min, green light illumination with a green laser $(532 \mathrm{~nm}, 22-25 \mathrm{~mW}$ at the cannula tip; Opto Engine) was applied continuously for $2 \mathrm{~min}$ through optical cannulae to activate eArch3 in the VH. After green light illumination, mice were left in context A for an additional $2 \mathrm{~min}$. Movement of the mice in the fear-conditioning chamber was recorded using a near-infrared camera and analyzed in real-time with Ethovision XT 11 software (Noldus). The freezing score was calculated as the percentage of time that the mice remained immobile. The bout length was $2 \mathrm{~s}$. Each mouse underwent the freeze test once per day for $3 \mathrm{~d}$ (days 8-10) and freezing scores ( 2 min bins) in the presence or absence of green light illumination were calculated separately on each test day and averaged. Fear conditioning and freeze testing were performed between 9:00 and 11:00 A.M. The temperature in the fear-conditioning chamber was $22-24^{\circ} \mathrm{C}$

For the c-Fos-immunostaining experiment (see Fig. $4 B$ ), mice were randomly assigned to three groups after virus injection surgery. On day 1 , mice in the fear-conditioning group were placed in context $\mathrm{A}$ in a fearconditioning chamber and given five US (a $0.5 \mathrm{~mA}$ electric foot shock for $2 \mathrm{~s}$ with $2 \mathrm{~min}$ intervals between the US). Mice in the context-only group were exposed to context A for 12 min without the US. On day 2, mice from both groups were placed in the same context A and freezing behavior was recorded for $12 \mathrm{~min}$. The freezing score was calculated as the percentage of time in which the mice remained immobile during the entire 12 min test session. Ninety minutes after the test session, the brain tissues from these mice were fixed for c-Fos immunohistochemistry. Mice in the home cage control group were left in home cages on days 1 and 2.

Histology and microscopic imaging. Acute brain slices (300 $\mu \mathrm{m}$ thick) were prepared with a vibratome (VT-1000S; Leica) and fixed in $4 \%$ paraformaldehyde-containing PBS ( $137 \mathrm{~mm} \mathrm{NaCl}, 2.7 \mathrm{~mm} \mathrm{KCl}, 11.9 \mathrm{~mm}$ phosphate, $\mathrm{pH} 7.4$ ) at $4^{\circ} \mathrm{C}$ overnight. After fixation, slices were washed 3 times in PBS containing $0.3 \%$ Triton X-100 (PBS-T) at room temperature for $10 \mathrm{~min}$ and permeabilized in $\mathrm{PBS}-\mathrm{T}$ at $4^{\circ} \mathrm{C}$ overnight. For Nissl staining, slices were incubated with Neurotrace fluoresent Nissl stain (1:40 diluted in PBS, $615 \mathrm{~nm}$ emission; Thermo Fisher Scientific) for $3 \mathrm{~h}$ at room temperature and washed in PBS-T 3 times for $10 \mathrm{~min}$ each time. After a final wash in PBS-T, Vectashield mounting medium with or without DAPI (Vector Laboratories) was applied to the slices, which were then covered with coverslips. Microscopic images were captured using the Leica TCS SP5 confocal system. Images captured with different fluorescent channels were merged using ImageJ software.

For fluorescent labeling of the recorded neurons (see Figs. 5E, 6E, F), neurons were loaded with the pipette solution containing $5 \mathrm{~mm}$ biocytin for $20 \mathrm{~min}$. The pipette was then withdrawn slowly and the brain slices were fixed at $4{ }^{\circ} \mathrm{C}$ overnight with $4 \%$ paraformaldehyde. After fixation, slices were washed with PBS-T three times for 15 min each time and incubated with a streptavidin-Alexa Fluor 568 conjugate $(20 \mu \mathrm{g} / \mathrm{ml}$ in PBS, Thermo Fisher Scientific) for $2 \mathrm{~h}$ at room temperature. The unbound streptavidin was then washed out with PBS three times for $20 \mathrm{~min}$ each and the slices were mounted onto slides. Images of the labeled neurons were taken using a Leica TSC SP5 confocal microscope and neuronal morphology and location within the MPFC or basal amygdala were analyzed.

Quantification of retrogradely labeled $v C A 1$ neurons. Using retrograde HSV, vCA1 neurons projecting to the $\mathrm{mPFC}$ and amygdala were labeled with eYFP (green) and mCherry (red), respectively. The proportions of vCA1 neurons projecting to the $\mathrm{mPFC}$ and/or amygdala were quantified based on the florescent labeling of their cell bodies (see Fig. 3). Confocal microscopic images of the CA1 area of the VH were taken with a $20 \times$ objective lens using the Leica TCS SP5 confocal system and Z-stacked using ImageJ software. For each mouse, images of 4-6 representative fields of the ventral CA1 hippocampal area were taken. The proportions of vCA1 neurons labeled with eYFP only, mCherry only, and eYFP + mCherry to the total number of all labeled vCA1 neurons were calculated in each field of the ventral CA1 hippocampal area and averaged for each mouse. Double-projecting vCA1 neurons were identified based on cell bodies labeled with both eYFP and mCherry. The proportion of double- projecting vCA1 neurons to all vCA1 neurons projecting to either the mPFC or amygdala was also calculated for each mouse.

$c$-Fos immunohistochemistry and analysis. Ninety minutes after the test session in context A on day 2 (see Fig. $4 B$ ), brain slices (100 $\mu$ m thick) were prepared with a vibratome and fixed in $4 \%$ paraformaldehydecontaining PBS at $4^{\circ} \mathrm{C}$ for $30 \mathrm{~min}$. After fixation, the slices were permeabilized in PBS-T at $4^{\circ} \mathrm{C}$ for $4 \mathrm{~d}$. Brain sections were then blocked with PBS containing $5 \%$ goat serum at $4^{\circ} \mathrm{C}$ for $1 \mathrm{~h}$. The slices were washed with PBS-T for 10 min and incubated with a rabbit anti-c-Fos antibody (1:500 in PBS-T, sc-52; Santa Cruz Biotechnology) at $4^{\circ} \mathrm{C}$ overnight. The slices were then washed with PBS-T three times for $10 \mathrm{~min}$ each time and incubated with goat anti-rabbit IgG antibody-Alexa Fluor 405 (1:200 in PBS-T, A-31556; Thermo Fisher Scientific) at $4^{\circ} \mathrm{C}$ overnight. The slices were then washed three times with PBS-T for $10 \mathrm{~min}$ each time and mounted onto glass slides for confocal microscopic imaging. For each mouse, $Z$-series confocal microscopic images of $4-6$ representative fields $\left(0.6 \mathrm{~mm}^{2}\right.$ area, $45 \mu \mathrm{m}$ thick) of the CA1 area of the $\mathrm{VH}$ and $Z$-stacked the images were captured using ImageJ software. vCA1 neurons projecting to either the mPFC (labeled with eYFP only) or amygdala alone (labeled with mCherry only), as well as double-projecting vCA1 neurons (labeled with both eYFP and mCherry), were identified. Then, the proportions of c-Fos + neurons (blue fluorescence) among all vCA1 neurons projecting to either the $\mathrm{mPFC}$ or amygdala alone or all double-projecting vCA1 neurons in each vCA1 field were calculated and the proportions averaged for each mouse.

Electrophysiology and photostimulation in brain slices. Four weeks after surgery, the mice were deeply anesthetized with $5 \%$ isoflurane and decapitated. Brains were dissected quickly and chilled in ice-cold artificial CSF (ACSF) containing the following (in $\mathrm{mm}$ ): $130 \mathrm{NaCl}, 2.5 \mathrm{KCl}, 2.5$ $\mathrm{CaCl}_{2}, 1 \mathrm{MgSO}_{4}, 1.25 \mathrm{NaH}_{2} \mathrm{PO}_{4}, 26 \mathrm{NaHCO}_{3}$, and 10 glucose with $95 \%$ $\mathrm{O}_{2}$ and $5 \% \mathrm{CO}_{2}$. Coronal brain slices containing the mPFC, amygdala, or $\mathrm{VH}(300 \mu \mathrm{m}$ thick) were prepared with a vibratome. After a $1 \mathrm{~h}$ recovery period, slices were placed in the recording chamber and perfused continuously with the ACSF at the rate of $1 \mathrm{ml} / \mathrm{min}$. For most of the whole-cell recordings, the patch electrodes (2-3 M $\Omega$ resistance) were filled with pipette solution containing the following (in $\mathrm{mM}$ ): 140 Cs-methanesulfonate, $5 \mathrm{NaCl}, 1 \mathrm{MgCl}_{2}, 10$ HEPES, 0.2 EGTA, $2 \mathrm{MgATP}, 0.5 \mathrm{NaGTP}$, and 5 QX 314 chloride (290 mOsm, adjusted to $\mathrm{pH} 7.3$ with $\mathrm{CsOH}$ ). Whole-cell patch-clamp recordings were performed at $30-32^{\circ} \mathrm{C}$ using a Multiclamp 700B amplifier, a Digidata 1550A digitizer, and Clampex 10 software (Molecular Devices). The membrane potential was held constant at -80 $\mathrm{mV}$ in voltage-clamp mode unless otherwise indicated. The liquid junction potential of $8.9 \mathrm{mV}$ was corrected. Series (access) resistance was $<20 \mathrm{M} \Omega$ and was not compensated. Offline data analysis was performed using the Clampfit 10 program (Molecular Devices).

A blue collimated light-emitting diode (LED) with a peak wavelength of $470 \mathrm{~nm}$ (M470L3; Thorlabs) was used for photostimulation of ChR2expressing axons (Cho et al., 2013). The LED was connected to the amplifier and digitizer through the LED driver (LEDD1B; Thorlabs). Brain slices in the recording chamber were illuminated through a $40 \times$ waterimmersion objective lens (LUMPLFLN 40XW; Olympus) and a 450-490 $\mathrm{nm}$ filter (Chroma). The illumination area was $0.17 \mathrm{~mm}^{2}$ and was centered at the soma of the neuron patched for recording. To evoke synaptic responses in the $\mathrm{mPFC}$ or amygdala by photostimulation of $\mathrm{VH}$ axons, slices were illuminated every $20 \mathrm{~s}$ with blue light pulses of $1 \mathrm{~ms}$ (light power density: $6-20 \mathrm{~mW} / \mathrm{mm}^{2}$ )

To stimulate the axon collaterals of double-projecting vCA1 neurons selectively and induce postsynaptic responses in the amygdala, canine adenovirus-2 (CAV-2) encoding Cre recombinase gene was injected into the mPFC and AAV-DIO-ChR2-eYFP into the VH (see Fig. 5A). In this experimental setup, CAV-2 is taken up by axon terminals in the mPFC and transported retrogradely to brain areas including the $\mathrm{VH}$ (Hnasko et al., 2006). Cre expression in mPFC-projecting vCA1 neurons then induces the recombination of a DIO, resulting in ChR2-eYFP expression (Atasoy et al., 2008). Because mPFC-projecting vCA1 neurons express ChR2, blue light illumination applied locally to the amygdala activated presynaptic axons of double-projecting $\mathrm{VH}$ neurons selectively, inducing EPSCs in BLA/BMA neurons. We used the same approach to stimulate 
selectively the axon collaterals of double-projecting vCA1 neurons in the mPFC (see Figs. 6A, 7A).

For the optogenetic inhibition experiment in brain slices (see Fig. $2 C-E), 140 \mathrm{~mm}$ Cs-methanesulfonate in the pipette solution was replaced with $150 \mathrm{~mm}$ K-gluconate without QX 314 added and the liquid junction potential of $15.5 \mathrm{mV}$ was corrected. A green LED with a peak wavelength of $565 \mathrm{~nm}$ (M565L3; Thorlabs) and a 540-580 nm filter (Chroma) was used to activate eArch3 (light power density: $10 \mathrm{~mW} / \mathrm{mm}^{2}$ ).

Reagents were prepared as stock solutions in water at 1000-fold concentrations and stored at $-20^{\circ} \mathrm{C}$. The reagents were purchased from Tocris Bioscience (NBQX, D-AP5, QX 314, and biocytin) or SigmaAldrich (ATP, GTP, and bicuculline methochloride).

Data analysis. Data are presented as means \pm SEM. For statistical comparisons, Student's $t$ test was used for paired samples (paired $t$ test), Welch's $t$ test for independent samples (unpaired $t$ test), and ordinary or repeated-measures ANOVA. For post hoc analysis, we used Bonferroni's simultaneous multiple comparisons. Statistical analysis was performed with Minitab 17 software (Minitab), and $p<0.05$ was considered statistically significant.

\section{Results}

\section{VH projects directly to the MPFC and basal amygdala}

To investigate how the hippocampus projects to the mPFC and amygdala, we transduced CA1 neurons of the $\mathrm{DH}$ and $\mathrm{VH}$ in mice with an AAV vector coding eYFP gene under the control of the CaMKII promoter (AAV-pCaMKII-eYFP, Fig. $1 A, B$ ). Four weeks later, eYFP expression in hippocampal neurons was identified with green fluorescence. The axonal fibers of eYFP-expressing hippocampal neurons were also identified with green fluorescence in coronal brain sections of the MPFC and amygdala. When AAV was injected into the $\mathrm{DH}$, we observed only weak eYFP-labeled projections in the $\mathrm{mPFC}$ and amygdala (Fig. 1A). However, when AAV was injected into the $\mathrm{VH}$, abundant eYFP-labeled projections were detected in the MPFC and amygdala (Fig. $1 B$ ). In the $\mathrm{mPFC}$, these projections were detected in both the prelimbic $(\mathrm{PL})$ and infralimbic (IL) divisions of the mPFC. Within the amygdala, projections from the $\mathrm{VH}$ were found predominantly in the basolateral and basomedial nuclei of the amygdala (BLA/BMA). These results suggest that the $\mathrm{MPFC}$ and basal amygdala receive strong monosynaptic inputs from the $\mathrm{VH}$ but only weak projections from the $\mathrm{DH}$.

To determine whether VH projections make functional synaptic contacts onto neurons in the mPFC and amygdala, we injected AAV coding ChR2 (H143R)-eYFP fusion gene under the control of the CaMKII promoter (AAV-pCaMKII-ChR2eYFP) (Boyden et al., 2005). Four weeks later, we examined the photostimulation-induced synaptic responses in these areas (Fig. $1 C-H)$. In the mPFC, short pulses of blue light illumination (1 $\mathrm{ms}$ duration) evoked robust EPSCs at $-80 \mathrm{mV}$ in voltage-clamp mode $(n=6$ cells; Fig. $1 C-E)$. EPSCs were also induced in the amygdala by photostimulation of $\mathrm{VH}$ projections expressing ChR2 (Fig. 1F, G), as reported previously (Hübner et al., 2014). Consistent with the projection pattern of the VH (Fig. 1B), the amplitude of the EPSCs was significantly larger in the basal amygdala (BLA/BMA) than in other nuclei of the amygdala [Fig. $1 \mathrm{H}$; main effect of subnuclei of the amygdala, $F_{(3,29)}=8.75, p<$ 0.001 , repeated-measures one-way ANOVA; $p<0.05$, BLA/BMA vs lateral nucleus of the amygdala (LA), intercalated cells, or central nucleus of the amygdala (CeA), post hoc Bonferroni's simultaneous multiple comparisons; $n=5-16$ cells per subnucleus]. These results indicate that the VH neurons make excitatory synaptic connections onto neurons in the $\mathrm{MPFC}$ and basal amygdala.

\section{Optogenetic inhibition of the $\mathrm{VH}$ prevents the recall of contextual fear memory}

Through its direct excitatory projections, the $\mathrm{VH}$ may regulate the neural activity of the mPFCand amygdala, which is necessary for the recall of contextual fear memory (Helmstetter and Bellgowan, 1994; Muller et al., 1997; Corcoran and Quirk, 2007; Goshen et al., 2011; Zhu et al., 2014). Therefore, we examined the role of the $\mathrm{VH}$ in the expression of conditioned fear using an in vivo optogenetic approach, which allows for examination of the effects of acute and reversible neural silencing on behavior in the same experimental subjects with a high degree of temporal precision. To silence neural activity in the $\mathrm{VH}$ in the experimental group (eArch3 group), we injected AAV encoding the eArch3 gene (AAV-eArch3) into the VH bilaterally (Chow et al., 2010; Mattis et al., 2011) and implanted bilateral optical cannulae into the CA1 area of the $\mathrm{VH}$ (Fig. $2 A, B$ ). eArch3 was expressed in vCA1 pyramidal neurons under the control of the CaMKII promoter. In eYFP control group, we injected AAV encoding the eYFP gene (AAV-eYFP) and implanted optical cannulae bilaterally into the vCA1 area. In brain slices from mice in the eArch3 group, green light illumination induced robust outward currents in voltage-clamp mode in vCA1 neurons expressing eArch3 (Fig. 2C). In current-clamp mode, the green light illumination induced eArch3-mediated hyperpolarization, which inhibited action potential firings in vCA1 neurons with high temporal precision $(n=5$ cells; Fig. $2 D, E)$. These results indicate that $\mathrm{VH}$ activity can be silenced in vivo using our optogenetic approach. Such effects were not observed in vCA1 neurons in the eYFP control group.

To investigate the role of the $\mathrm{VH}$ in conditioned fear expression, we examined how acute silencing of $\mathrm{VH}$ activity affected the recall of fear memory after contextual fear conditioning. After being habituated to handling and optical cable connection for $6 \mathrm{~d}$ (days 1-6), mice in both the eArch3 and eYFP groups were fear conditioned in context $\mathrm{A}$ on the training day (day 7) by receiving the US (electric foot shocks, $0.5 \mathrm{~mA}, 2 \mathrm{~s}$ duration; Fig. $2 F$ ). After contextual fear conditioning, mice were tested for conditioned fear responses (freezing behavior) in context A once per day for 3 successive days (days $8-10)$. On the test days, freezing behavior was monitored in context A. During the first $2 \mathrm{~min}$ of the behavioral testing, mice in both the eArch3 and eYFP groups displayed robust freezing behavior in context A (Fig. 2G). After the baseline measurement of freezing behavior, illumination with a green laser was applied to the $\mathrm{VH}$ through the optical cannulae for $2 \mathrm{~min}$ in both groups, followed by behavioral monitoring without illumination for an additional $2 \mathrm{~min}$. The freezing score was calculated for each 2 min session. Repeated-measures two-way ANOVA revealed a significant interaction between laser (on vs off) and group (eArch3 vs eYFP) $\left(F_{(1,10)}=6.67, p<0.05\right)$. Post hoc comparisons revealed that the freezing behavior in the eArch3 group was significantly reduced by green laser illumination (Fig. 2G,H; $p<0.01$, laser on vs laser off, Bonferroni's simultaneous multiple comparisons; $n=5$ mice), whereas the same green laser illumination did not affect freezing behavior in the eYFP control group (Fig. $2 G, H ; p=0.49 ; n=7$ mice), indicating that the effect of green laser illumination on freezing behavior was dependent upon eArch3 expression in the $\mathrm{VH}$. These results suggest that $\mathrm{VH}$ activity is necessary for the recall of contextual fear memory.

\section{Some ventral CA1 hippocampal neurons project to both the mPFC and amygdala with axon collaterals}

The VH may contribute to the recall of contextual fear memory by conveying context representations to the mPFC and amygdala. Therefore, we determined the proportions of $\mathrm{VH}$ neurons projecting to either the mPFC or amygdala using dual retrograde viral tracing with HSV, which is taken up by axon terminals and transported 
A

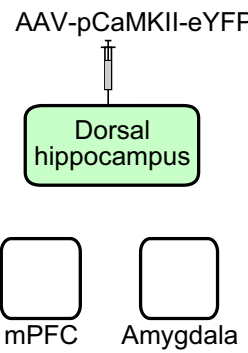

\section{B}

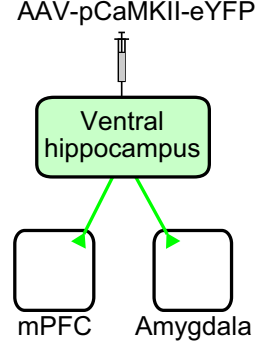

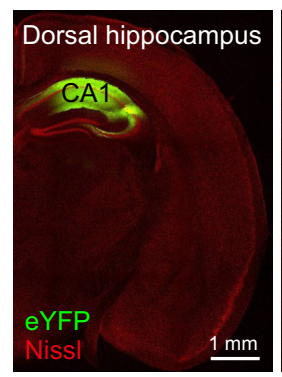
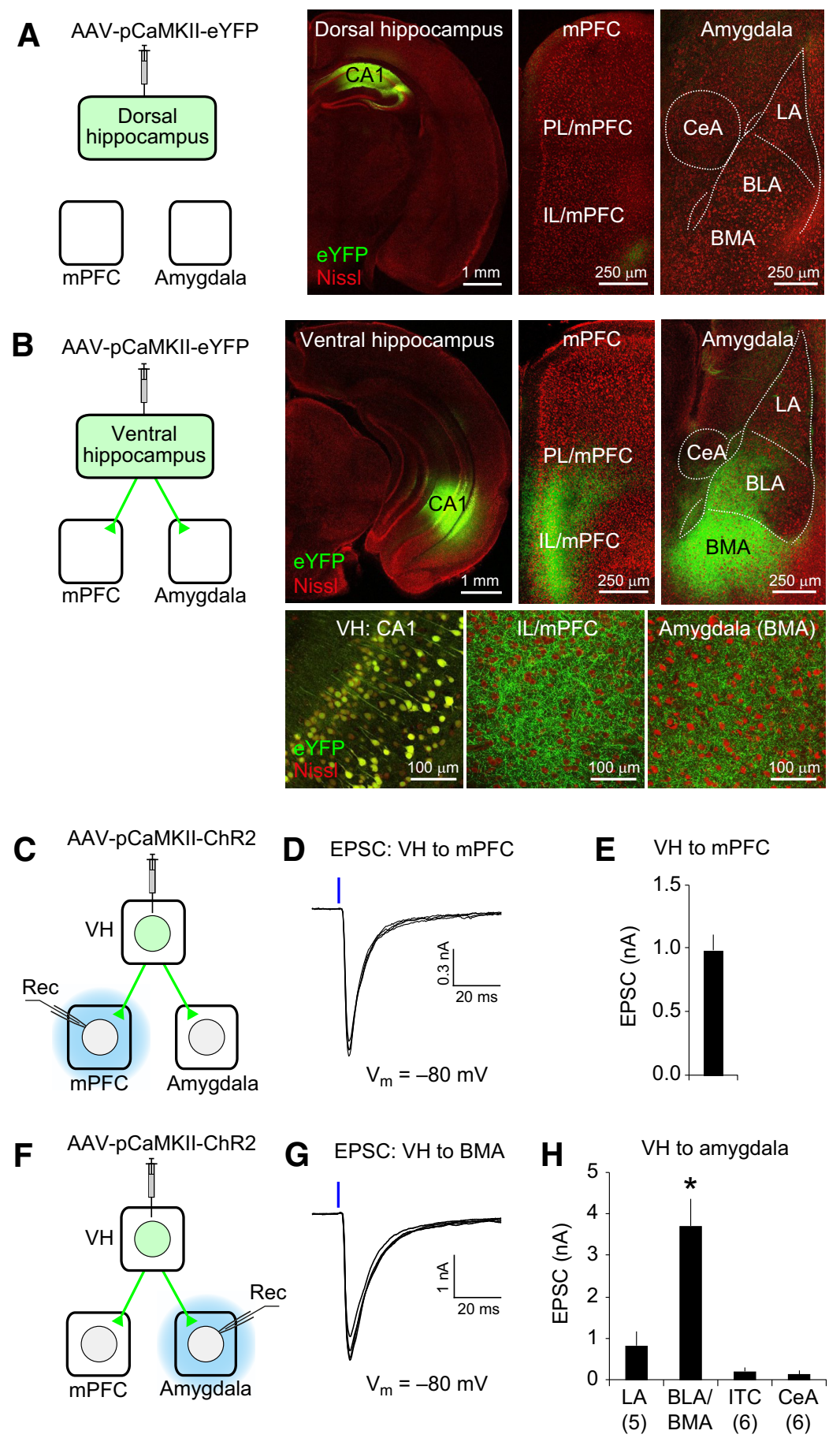

(16)

Figure 1. The VH projects directly to the MPFC and basal amygdala. A, Left, AAV-pCaMKII-eYFP was injected into the CA1 area of the DH. eYFP was expressed in dorsal CA1 neurons under the control of the CaMKII promoter (pCaMKII). Right, eYFP expression in the DH, $m P F C$, and amygdala (eYFP in green; fluorescent Nissl stain in red). Few eYFP-expressing projections from the DH were detected in the mPFC and amygdala. LA, BLA, BMA, and CeA are the lateral, basolateral, basomedial and central nucleus of the amygdala, respectively. B, AAV-pCaMKII-eYFP was injected into the VH. Confocal microscopic images show eYFP expression (green fluorescence) in the VH and eYFP-labeled VH projections in the mPFC and amygdala at a low (top) or high magnification (bottom). Note eYFP-labeled cell bodies in the CA1 area of the VH and eYFP-labeled axons in the IL/mPFC and BMA in the magnified images (bottom). C, Experimental setup for $\boldsymbol{D}$ and $\boldsymbol{E}$. AAV-pCaMKII-ChR2-eYFP was injected into the VH. ChR2-eYFP is expressed in vCA1 neurons under the control of the CaMKII promoter (pCaMKII). Blue light illumination was applied locally to the mPFC in brain slices (blue) to stimulate VH projections expressing ChR2 and induce the postsynaptic responses recorded in the mPFC (Rec). D, Representative traces of EPSCs in the VH-mPFC pathway. A blue vertical bar indicates blue light illumination $\left(20 \mathrm{~mW} / \mathrm{mm}^{2}, 1 \mathrm{~ms}\right.$ duration) onto ChR2-expressing VH projections in the mPFC. $\boldsymbol{E}$, Average peak amplitude of EPSCs in the VH-mPFC pathway recorded as described in $\boldsymbol{D}$. $n=6$ cells. $\boldsymbol{F}$, Experimental setup for $\boldsymbol{G}$ and $\boldsymbol{H}$. AAV-pCaMKII-ChR2-eYFP was injected into the VH. Blue light illumination was applied locally to the amygdala in brain slices (blue) to stimulate ChR2-expressing VH projections and induce the postsynaptic responses recorded in the amygdala (Rec). G, Representative traces of EPSCs in the VH-amygdala pathway. EPSCS to the soma. We injected HSV coding eYFP (HSV-eYFP) into the mPFC and HSV coding mCherry gene (HSV-mCherry) into the basal amygdala, which includes the BLA and BMA (Fig. 3A). The cell bodies of VH neurons projecting to the $\mathrm{MPFC}$ and amygdala were labeled with eYFP and mCherry, respectively. Consistent with the results of our anterograde viral tracing experiments (Fig. $1 A, B$ ), neurons projecting to the $\mathrm{MPFC}$ and amygdala were found in the $\mathrm{VH}$, but were not detected in the $\mathrm{DH}$ (Fig. $3 B, C$ ). VH neurons projecting to the $\mathrm{mPFC}$ and amygdala were found in the CA1 area and subiculum of the VH (Fig. 3B), which was consistent with previous reports (Ishikawa and Nakamura, 2006; Jin and Maren, 2015). Most of the labeled vCA1 neurons expressed either eYFP or mCherry alone, indicating that they project to either the MPFC or amygdala alone (mPFC projectors: $43.7 \pm 2.6 \%$ of all labeled vCA1 neurons; amygdala projectors: $47.3 \pm 2.6 \%$; mean \pm SEM; $n=22$ mice; Fig. $3 D$ ). Surprisingly, $9.0 \pm 0.6 \%$ of all labeled vCA1 neurons were both eYFP and mCherry positive (Fig. $3 B, D$ ), suggesting that they project to both the $\mathrm{mPFC}$ and amygdala. We also found that $18.0 \pm 1.5 \%$ of mPFC-projecting vCA1 neurons also projected to the amygdala and $16.6 \pm 1.2 \%$ of amygdala-projecting vCA1 neurons also projected to the mPFC with axon collaterals (mean \pm SEM, $n=22$ mice; Fig. $3 E-G$ ). These results suggest that a significant proportion of $\mathrm{vCA} 1$ neurons have long-range projections to both the $\mathrm{mPFC}$ and basal amygdala.

Double-projecting vCA1 neurons are activated preferentially during context encoding and the recall of contextual fear memory

We next examined the role of vCA1 neurons projecting to the $\mathrm{mPFC}$ and/or amygdala in context encoding and contextual fear expression using c-Fos immunohistochemistry combined with retrograde tracing. We injected HSVeYFP and HSV-mCherry into the mPFC and amygdala, respectively, to label vCA1 neurons projecting to these areas (Fig. $4 A)$. After surgery, mice in the fearconditioning group received electric foot shocks $(0.5 \mathrm{~mA}, 120 \mathrm{~s}$ interval) in a novel context $\mathrm{A}$, whereas the mice in the

$\leftarrow$

were induced as described in $\boldsymbol{D}$ and recorded in a BMA neuron. $H$, Quantification of the peak amplitudes of EPSCS evoked by stimulation of $\mathrm{VH}$ projections and recorded in different nuclei of the amygdala. The numbers in the parentheses are the number of neurons examined for each nucleus of the amygdala. ${ }^{*} p<0.05, B L A / B M A$ versus $L A$, intercalated cells (ITC), or CeA. Error bars indicate SEM. 
A

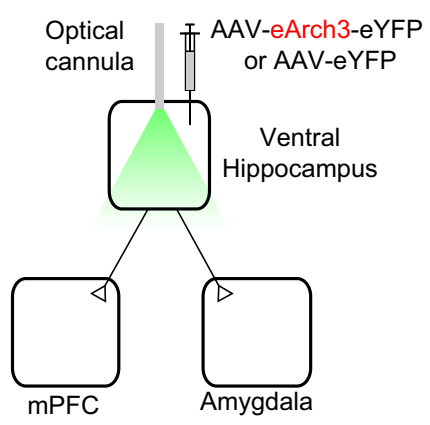

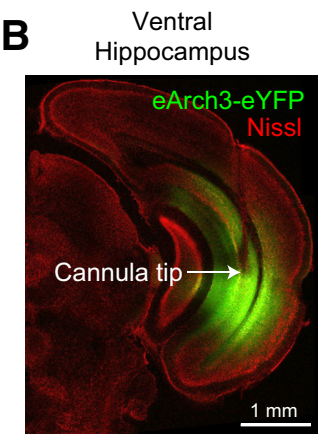
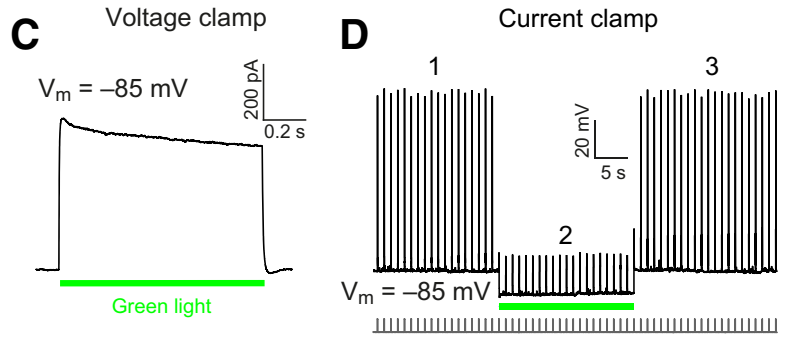
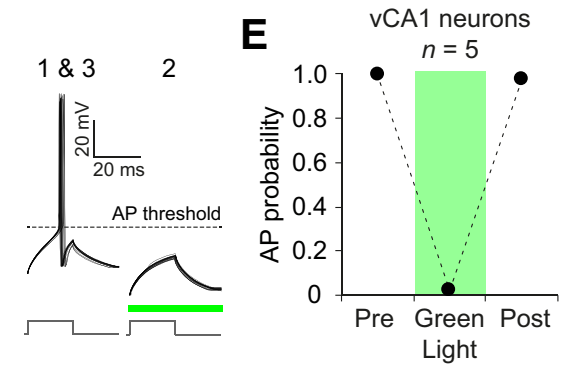
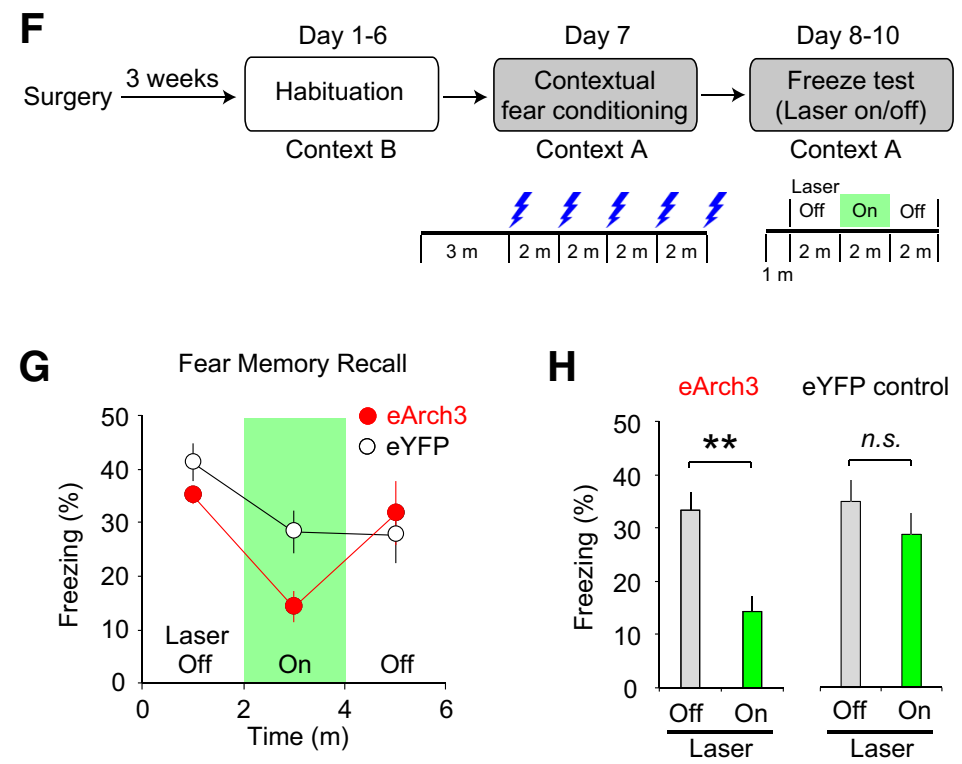

Figure 2. Optogenetic silencing of the VH prevents the recall of contextual fear memory. $A, A A V-e A r c h 3-e Y F P$ was injected and optical cannulae were implanted bilaterally into the VH to silence VH activity in vivo (eArch3 group, $n=5$ mice). AAV-eYFP was injected into the VH in the eYFP control group ( $n=7$ mice). $\boldsymbol{B}$, Microscopic image showing eArch-eYFP expression (green fluorescence) and the cannula implantation site (arrow). Red fluorescence indicates Nissl stain. C, Representative trace of eArch3-mediated currents induced by green light illumination $\left(565 \mathrm{~nm}, 10 \mathrm{~mW} / \mathrm{mm}^{2}\right.$, green horizontal bar) and recorded in voltage-clamp mode at $-85 \mathrm{mV}$ in an eArch3-expressing vCA1 neuron in a brain slice. $\boldsymbol{D}$, Left, Representative trace recorded in current-clamp mode showing the inhibition of action potentials (APs) by green light illumination (10 mW/mm², green horizontal bar), which induced hyperpolarization in eArch3-expressing vCA1 neurons in brain slices. Right, Traces showing AP firings and their inhibition by green light illumination in a different time scale. The baseline membrane potential $\left(V_{\mathrm{m}}\right)$ was $\sim-85 \mathrm{mV}$. APs were induced by square pulses of current injections ( $150 \mathrm{pA}, 20 \mathrm{~ms}$ duration) at $1 \mathrm{~Hz}$ frequency as indicated below the traces. $E$, Quantification of AP firing probability in the presence or absence of green light illumination. AP firing was inhibited almost completely by green light illumination activating eArch3. $n=5$ eArch3-expressing CA1 neurons. $F$, Experimental setup. After surgery, mice were habituated to handling and optical cable connection in context B for $6 \mathrm{~d}$ (days 1-6) and fear conditioned in context A on day 7. On days 8 -10, freezing behavior was monitored in context A in the presence and absence of green light illumination onto the VH. G, Time course of freezing behavior in context A after contextual fear conditioning in the eArch3 ( $n=5$ mice, red closed circle) and eYFP groups ( $n=7$ mice, black open circles). After a $1 \mathrm{~min}$ acclimatization period and baseline recording of freezing behavior for $2 \mathrm{~min}$, green light illumination was applied to the VH through bilateral optical cannulae for 2 min (green area within the graph). The freezing score was calculated as the percentage of time in which the mice remained immobile and the bout length was $2 \mathrm{~s}$. For each mouse, freezing score (\%) was calculated on each test day (days $8-10$ ) and averaged for each time bin. $\boldsymbol{H}$, Quantification of freezing behavior in context A. The recall of contextual fear memory was inhibited by optogenetic silencing of the VH in the eArch3 group, but not in the eYFP control group. Freezing scores on days $8-10$ were averaged for each mouse. Freezing behavior was significantly reduced by green laser illumination in the eArch3 group $\left({ }^{* *} p<0.01\right.$, laser on vs laser off), whereas it was not affected by laser illumination in the eYFP control group (n.S., not significant, laser on vs laser off). Error bars indicate SEM.

context-only group were exposed to context A without an aversive stimulus (Fig. $4 B$ ). After $24 \mathrm{~h}$, these mice were exposed to context $\mathrm{A}$ again and tested for conditioned fear responses. On the test day, mice in the fear-conditioning group displayed robust freezing behavior in context $\mathrm{A}$, whereas mice in the context-only group did not $\left(t_{(5)}=3.86, p<0.05\right.$, unpaired $t$ test; $n=6$ mice per group; Fig. $4 C$ ), indicating the expression of contextual fear memory in the fear-conditioning group. Ninety minutes after 

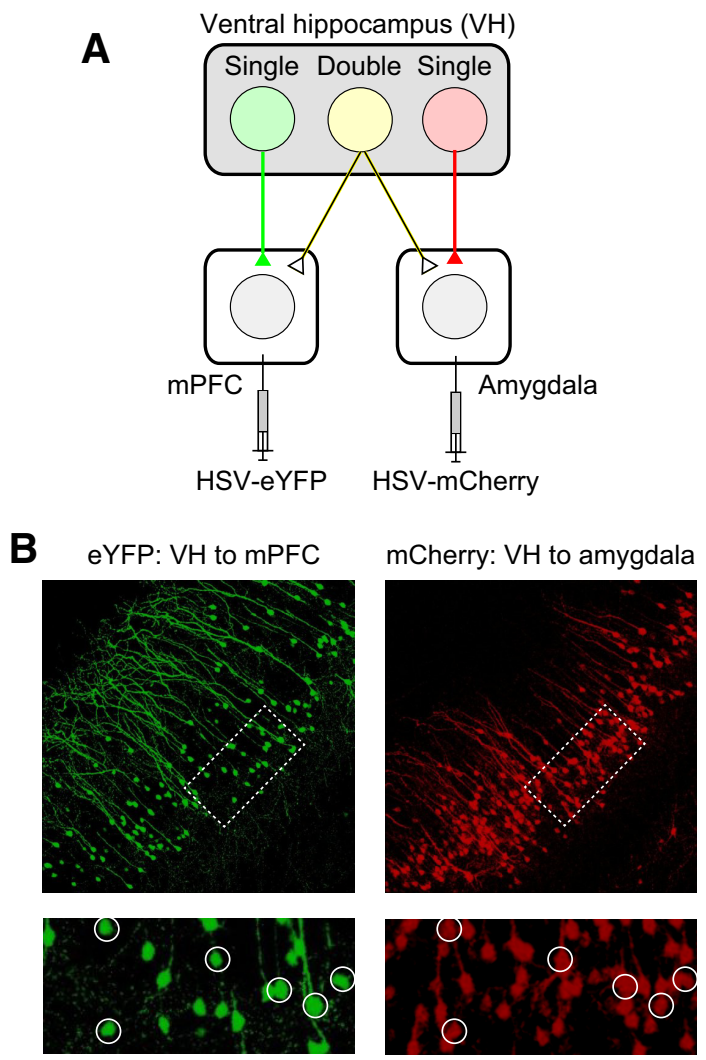

\section{D}

All labeled
vCA1 neurons

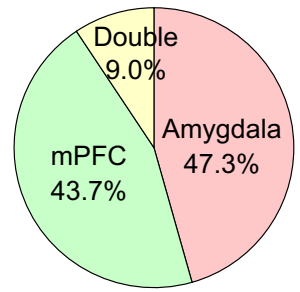

$n=22$ mice
E

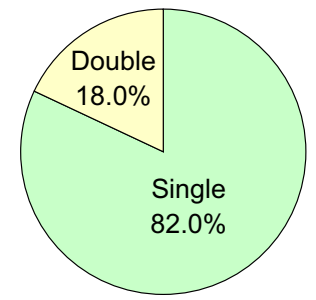

VH: bregma $-3.2 \mathrm{~mm}$
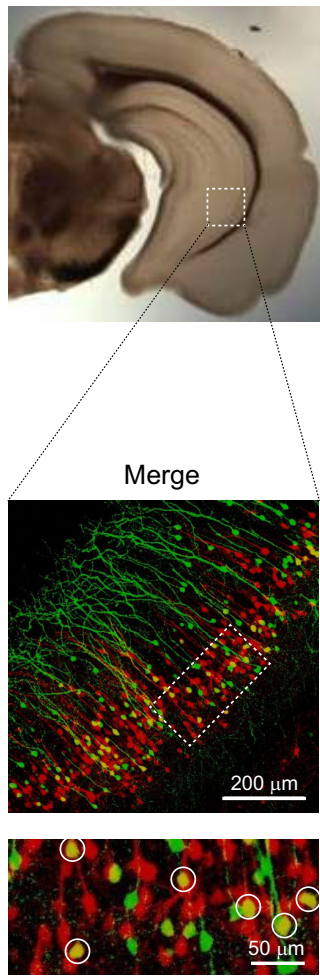

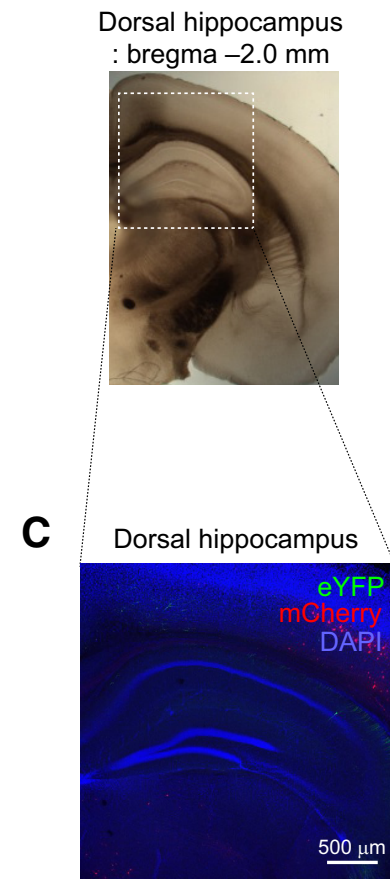

Dorsal hippocampus

Ventral hippocampus

F vCA1 to amygdala

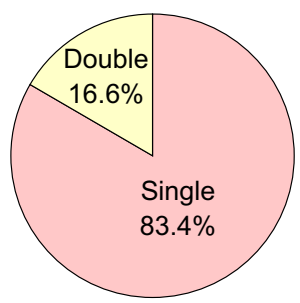

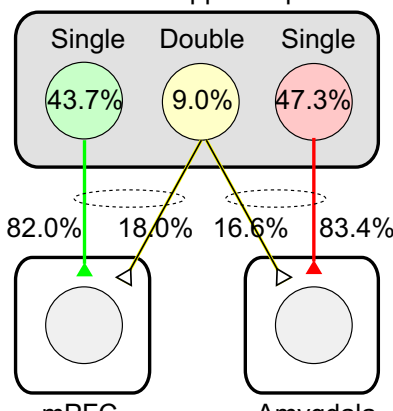

mPFC

G

Amygdala

Figure 3. Someventral CA1 hippocampal neurons project to both the mPFC and amygdala. $A$, Left, Retrograde viral tracers HSV-eYFP and HSV-mCherry were injected into the mPFC and basal amygdala (BLA and BMA), respectively. Right, Microscopic images of the coronal brain sections containing the DH and VH. Dotted squares indicate the areas magnified in $\boldsymbol{B}$ and $\boldsymbol{C}$. $\boldsymbol{B}$, Microscopic images showing vCA1 neurons projecting to the mPFC (eYFP-positive, green fluorescence, left) and to the amygdala ( $m$ Cherry-positive, red fluorescence, middle). These images are overlaid in the right panels and show double-projecting vCA1 neurons expressing both eYFP and mCherry (yellow). The pyramidal cell layer of the vCA1 area (dotted squares) in each fluorescence channel is shown at a higher magnification in the bottom and doubleprojecting vCA1 neurons are indicated with circles. C, Microscopic image of the DH from a mouse receiving injections of HSV-eYFP and HSV-mCherry into the mPFC and amygdala, as described in $A$. Note the lack of eYFP- and mCherry-positive neurons in the DH. Blue fluorescence represents DAPI staining. D, Pie chart showing the average proportion of $\mathrm{VCA}$ 1 neurons projecting to the mPFC and/or amygdala of all labeled $\operatorname{VCA} 1$ neurons $(n=22 \mathrm{mice})$. $\boldsymbol{E}$, Pie chart showing the average proportion of double-projecting vCA1 neurons of all CA1 neurons projecting to the mPFC $(n=22$ mice). $\boldsymbol{F}$, Piechart showing the average proportion of double-projecting vCA1 neurons of all CA1 neurons projecting to the basal amygdala ( $n=22$ mice). $\mathbf{G}$, Diagram summarizing the results in $\boldsymbol{D}-\boldsymbol{F}$.

context exposure on the test day, brain tissues from these mice were fixed for c-Fos immunohistochemistry. Mice in the home cage control group were left in home cages until brain fixation. As in the previous experiments, we identified vCA1 neurons projecting to the MPFC and amygdala with eYFP and mCherry fluorescence (Fig. 4D,E). There were no significant differences in the proportions of vCA1 neurons labeled with eYFP and/or mCherry between the behavioral groups (main effect of behavioral group: $F_{(2,30)}=0.29, p=0.75$; main effect of eYFP/mCherry labeling: $F_{(2,30)}=59.29, p<0.001$; group $\times$ labeling interaction: $F_{(4,30)}=$ $1.36, p=0.27$; repeated-measures two-way ANOVA; $n=6$ mice per group), suggesting that the proportions of vCA1 neurons projecting to the mPFC and/or amygdala were not different between the behavioral groups.

We next compared the proportion of c-Fos expression in vCA1 neurons of different projection types between the behavioral groups. A repeated-measures two-way ANOVA with factors of projection type (mPFC-projecting, amygdala-projecting, or double-projecting) and group (home cage group, context-only group, or fear-conditioning group) revealed a significant group $\times$ projection type interaction $\left(F_{(4,30)}=3.03, p<0.05 ; n=6\right.$ mice per group; Fig. $4 F)$. The c-Fos expression pattern of vCA1 neurons did not differ between the context-only and fear-conditioning groups (main effect of behavioral group: $F_{(1,20)}=0.00, p=0.96$; 

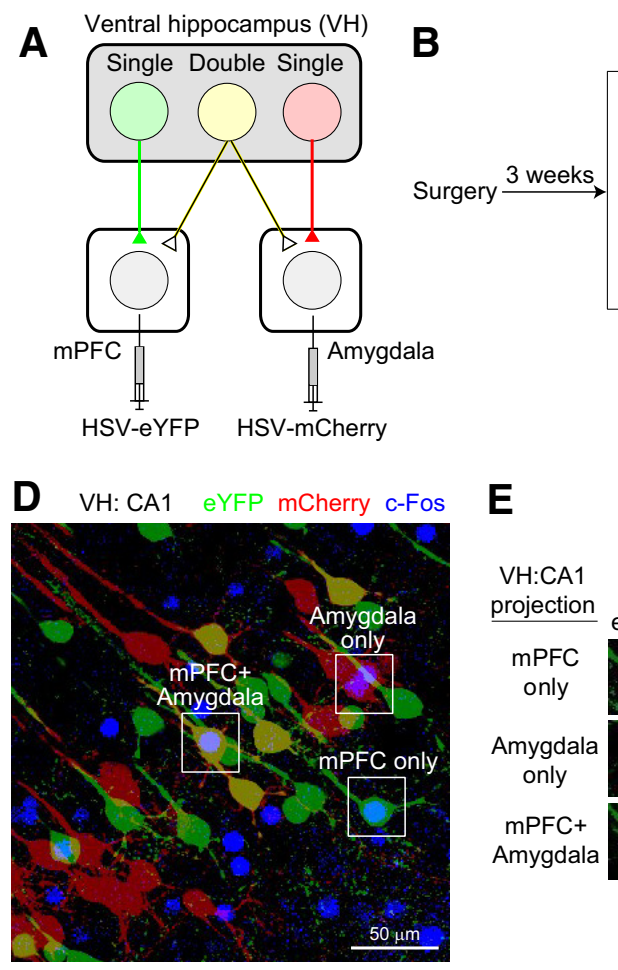

E

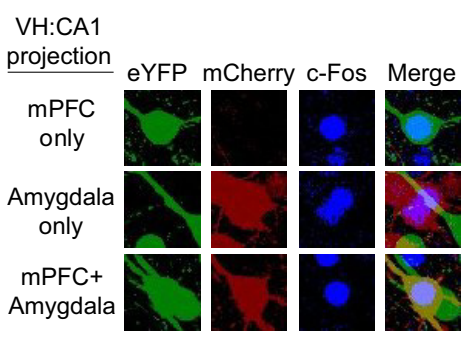

C

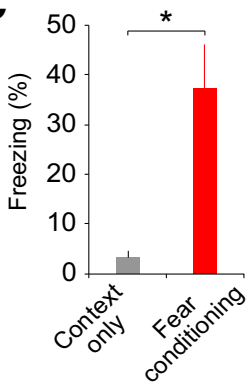

F $\quad \square$ Home cage

Fear conditioning

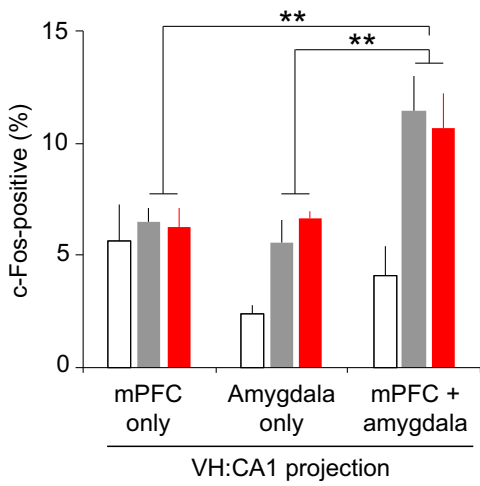

Figure 4. Double-projecting vCA1 neurons are activated preferentially during context exposure and the recall of contextual fear memory. $A$, Retrograde HSV-eYFP and HSV-mCherry were injected into the $\mathrm{mPFC}$ and basal amygdala to label vCA1 neurons projecting to these areas. $B, 0$ the training day, mice in the fear-conditioning group received 5 electric foot shocks ( $0.5 \mathrm{~mA}, 2 \mathrm{~s}$ duration) in a novel context $\mathrm{A}$, whereas the mice in the context-only group were exposed to the context without an aversive stimulus. After $24 \mathrm{~h}$, these mice were tested for freezing behavior in context $\mathrm{A}$. Ninety minutes after the behavioral test, brain tissues from these mice were fixed for $c$-Fos immunostaining. Mice in the home cage control group were left in home cages until brain fixation. $n=$ 6 mice per group. C, Quantification of freezing behavior in context A on the test day in the context-only and fear-conditioning groups. ${ }^{*} p<0.05$. D, Microscopic image of $v C A 1$ pyramidal neurons. VCA1 neurons projecting to the MPFC and amygdala were labeled with eYFP (green) and mCherry (red), respectively. Double-projecting vCA1 neurons were labeled with both eYFP and mCherry (mPFC + amygdala, yellow). Blue fluorescence indicates c-Fos expression in vCA1 neurons. Squares indicate the areas magnified in E.E, Microscopic images showing examples of c-Fos-positive vCA1 neurons projecting to the $\mathrm{mPFC}$ and/or amygdala in different fluorescent channels. $F$, Quantification of the proportion of c-Fos-positive vCA1 neurons projecting to the mPFC and/or amygdala for each behavioral group. When the mice were exposed to the context or recalled contextual fear memory, a greater proportion of double-projecting vCA1 neurons expressed c-Fos compared with vCA1 neurons projecting to either the mPFC or amygdala alone $\left({ }^{* *} p<0.01\right)$. Error bars indicate SEM.

main effect of projection type: $F_{(2,20)}=15.23, p<0.001$; group $\times$ projection type interaction: $F_{(2,20)}=0.42, p=0.66$; Fig. $\left.4 F\right)$. Therefore, we collapsed these groups to simplify the analysis and performed a repeated-measures two-way ANOVA, which also revealed a significant group $\times$ projection type interaction $\left(F_{(2,32)}=\right.$ $5.92, p<0.01)$. Post hoc analysis indicated that a greater proportion of double-projecting vCA1 neurons expressed c-Fos compared with the single-projecting vCA1 neurons when the mice were exposed to the context or recalled contextual fear memory $(p<0.01$, Bonferroni's simultaneous multiple comparisons; Fig. $4 F)$. However, there was no difference in c-Fos-positive proportion between single- and double-projecting vCA1 neurons in the home cage group ( $p=1.00$; Fig. $4 F$ ). These results suggest that double-projecting vCA1 neurons are activated preferentially during context exposure and the recall of contextual fear memory rather than vCA1 neurons projecting to either the mPFC or amygdala alone.

\section{Double-projecting vCA1 neurons make excitatory synapses} onto principal neurons in the basal amygdala

Our results suggest that double-projecting vCA1 neurons may contribute to the encoding of context representations, which are conveyed to the mPFC and amygdala for contextual fear responses. Therefore, we investigated whether the projections of these vCA1 neurons formed synaptic contacts onto neurons in the amygdala and mPFC. To this end, we developed an optogenetic approach to stimulate selectively the axon collaterals of double-projecting vCA1 neurons to induce postsynaptic responses in the amygdala or mPFC (Figs. 5, 6). We first injected CAV-2 encoding the Cre recombinase gene into the mPFC. We also injected AAV-DIO-ChR2-eYFP into the CA1 area of the $\mathrm{VH}$ (Fig. 5A). In this experimental setup, CAV-2 is taken up by axons terminals in the $\mathrm{mPFC}$ and transported retrogradely to brain areas including the VH (Hnasko et al., 2006). Cre expression in $\mathrm{mPFC}$-projecting vCA1 neurons then induces the recombination of a DIO, resulting in ChR2-eYFP expression (Atasoy et al., 2008). We identified the mPFC-projecting vCA1 neurons with eYFP expression (Fig. 5B) and observed eYFP-labeled axons of vCA1 neurons within the mPFC (Fig. 5C). eYFP-labeled axons were also detected in the amygdala, predominantly in the basal amygdala (BLA/BMA), where neither CAV-2 nor AAV was injected (Fig. 5D). This finding suggests that they originated from vCA1 neurons projecting to both the $\mathrm{MPFC}$ and amygdala, which is consistent with the results of our retrograde viral tracing experiments (Fig. 3).

We next performed whole-cell patch-clamp recordings in principal neurons of the basal amygdala (BLA/BMA) in brain slices from these mice (Fig. 5E). Because mPFC-projecting vCA1 
A

CAV2-Cre

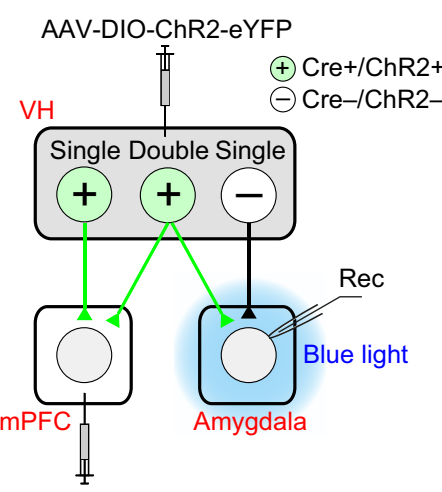

B
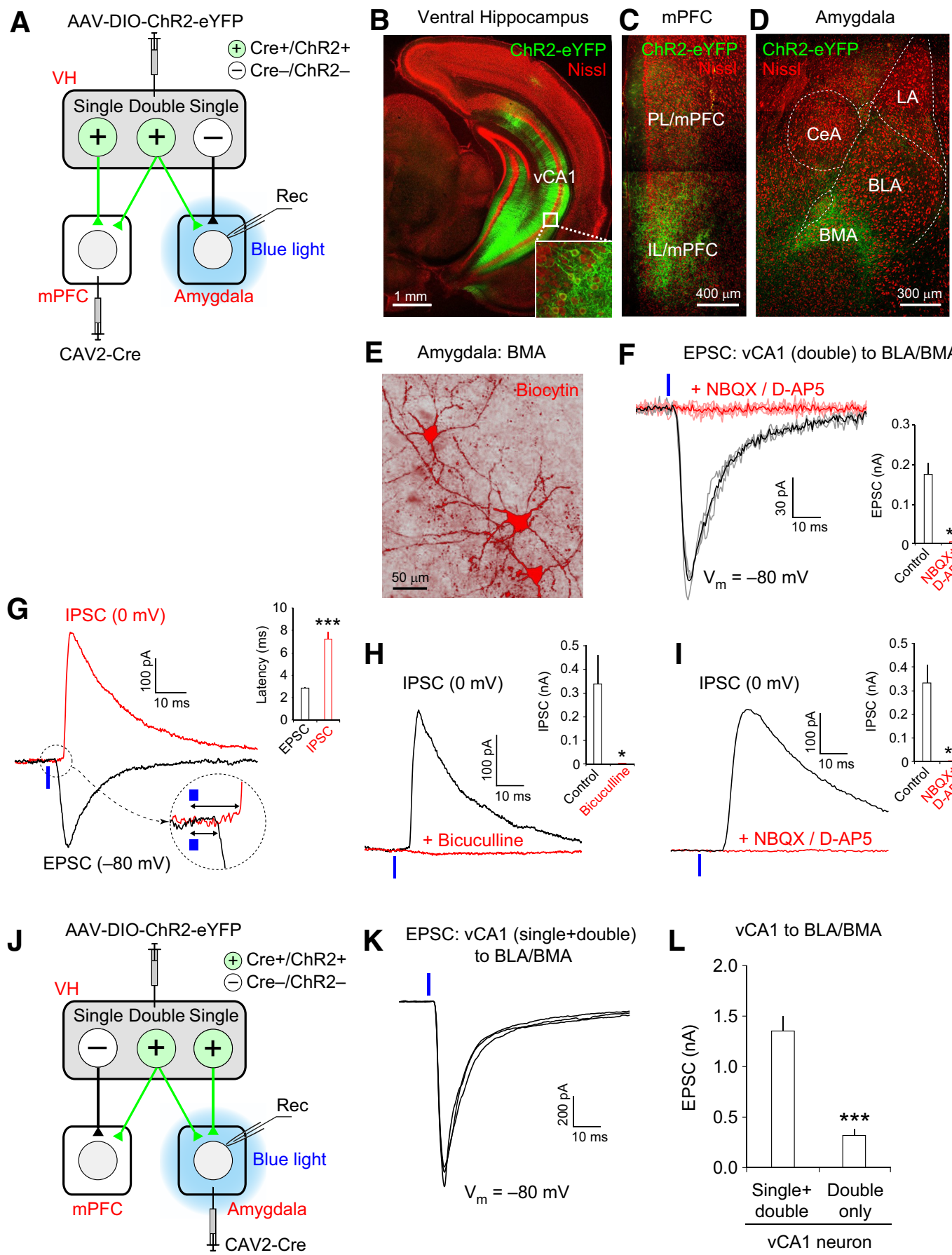

E
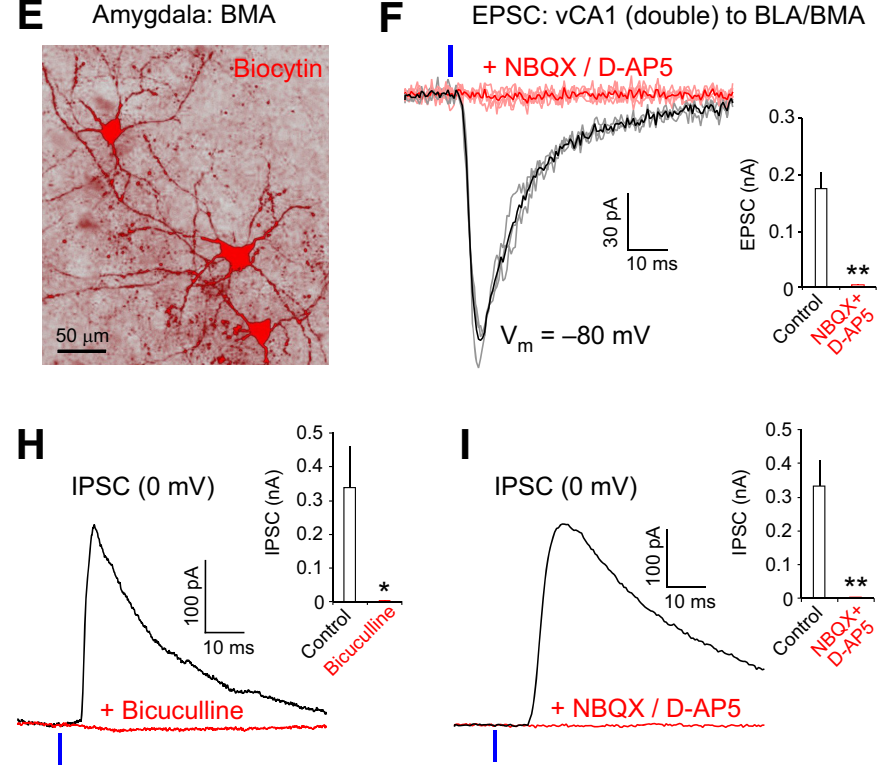

$\mathbf{F}$
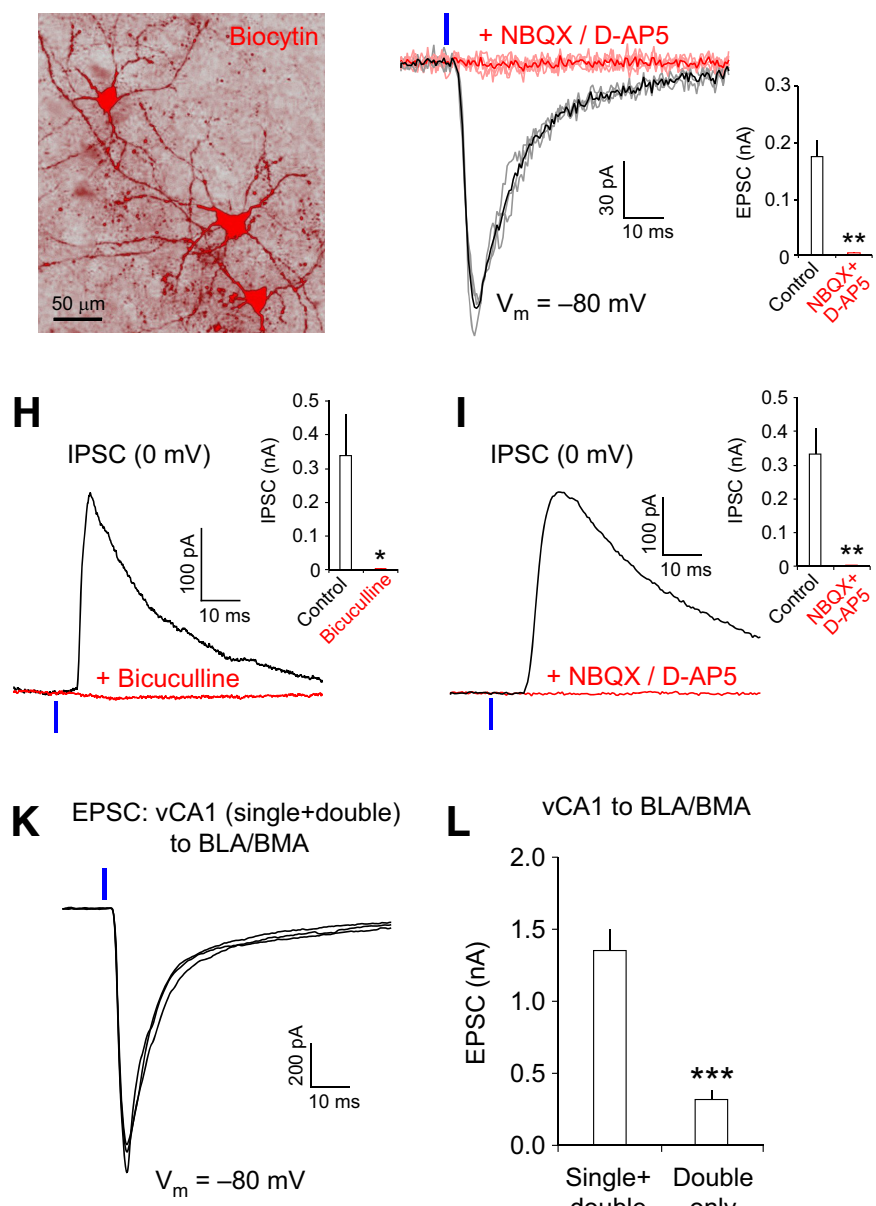

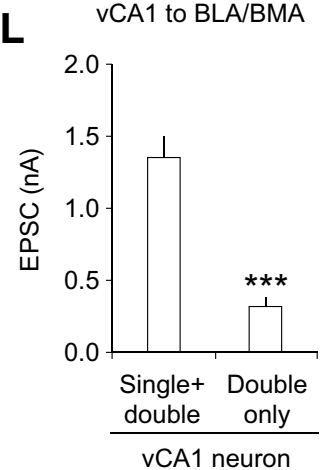

Figure 5. Double-projecting vCA1 neurons make excitatory synapses onto BLA/BMA neurons. $\boldsymbol{A}$, Experimental setup for $\boldsymbol{B}$ - $\boldsymbol{I}$. Retrograde CAV2-Cre was injected into the mPFC, and AAV-DI0ChR2-eYFP was injected into the CA1 area of the VH, resulting in the expression of Cre recombinase (Cre + ) and ChR2-eYFP (ChR2 +$)$ in vCA1 neurons projecting to the mPFC. Local blue light illumination onto the amygdala activated the axon collaterals of double-projecting vCA1 neurons, inducing postsynaptic responses in the amygdala (Rec). vCA1 neurons projecting to the amygdala, but not to the mPFC, did not express (re or ChR2 (Cre - /ChR2 - ) and their axons were not activated by local photostimulation. $\boldsymbol{B}-\boldsymbol{D}$, Confocal microscopic images of the VH, mPFC, and amygdala taken 4 weeks after viral injection surgery, as described in $\boldsymbol{A}$. Green fluorescence represents eYFP and red fluorescence represents Nissl stain. $\boldsymbol{B}$, Green fluorescence in the VH indicates eYFP-labeled vCA1 pyramidal neurons projecting to the $\mathrm{mPFC}$ (inset is a magnified image of vCA1 neurons). $C$, Green fluorescence in the mPFC indicates the eYFP-labeled axons of mPFC-projecting vCA1 neurons. $D$, Green fluorescence in the amygdala indicates axons from vCA1 neurons projecting to both the mPFC and basal amygdala (BLA/BMA).E, Representative image showing three principal neurons in the BMA loaded with biocytin (red fluorescence) during the whole-cell patch-clamp recording. These BMA neurons were labeled with streptavidin-Alexa Fluor 568 after recording. $\boldsymbol{F}$, Left, Representative traces of EPSCs induced by photostimulation of the axons of double-projecting vCA1 neurons and recorded in a BMA neuron. EPSCs were evoked by photostimulation (470 $\mathrm{nm}$ blue LED, $20 \mathrm{~mW} / \mathrm{mm}^{2}, 1 \mathrm{~ms}$ duration, $0.05 \mathrm{~Hz}$ stimulation frequency, blue vertical bar) applied locally onto the amygdala in brain slices and recorded at $-80 \mathrm{mV}$ in voltage-clamp mode. The black trace is the average of individual EPSCS (gray traces) under control conditions. EPSCs were completely blocked by the application of NBQX (10 $\mu \mathrm{M})$ and D-AP5 (50 $\mu \mathrm{M})$, shown as a red trace, which is the average of individual EPSCs (pink traces). Right, Quantification of EPSC inhibition by NBQX and D-AP5 (** $p<0.01, n=7$ cells). G, Representative traces of the EPSC (black trace) and IPSC (red trace) recorded in a BMA neuron. Synaptic responses were induced by photostimulation of double-projecting vCA1 neurons (blue vertical bars), as described in $\boldsymbol{F}$. EPSCs and IPSCs were recorded at -80 and $0 \mathrm{mV}$, respectively, in voltage-clamp mode in the same BMA neuron. These traces are the average of three individual traces of EPSCS or IPSCs. The initial portion of the postsynaptic responses is magnified in a dotted circle. Inset, Graph showing the average synaptic delay of EPSCS and IPSCs. The onset of IPSCS was more delayed than EPSCS (*** $p<0.001, n=12$ cells). $\boldsymbol{H}$, Representative traces of IPSCs induced and recorded as described in G. The black trace is IPSCs recorded under control conditions. IPSCS were completely blocked by the application of (Figure legend continues.) 
neurons express ChR2, blue light illumination applied locally to the amygdala activated presynaptic axons of double-projecting $\mathrm{VH}$ neurons selectively, inducing EPSCs in BLA/BMA neurons at $-80 \mathrm{mV}$ in voltage-clamp mode (Fig. $5 F$ ). These EPSCs were inhibited completely by the application of AMPA- and NMDAtype glutamate receptor antagonists $\left(\mathrm{NBQX}+\mathrm{D}-\mathrm{AP} 5 ; t_{(6)}=\right.$ 5.96, $p<0.01$, paired $t$ test; $n=7$ cells; Fig. $5 F$ ), indicating that the excitatory neurotransmitter glutamate mediated the EPSCs. We also investigated whether double-projecting vCA1 neurons activated local inhibitory interneurons and induced feedforward inhibition in principal neurons of the BLA/BMA. At $0 \mathrm{mV}$ in voltage-clamp mode, photostimulation of the axon collaterals of double-projecting vCA1 neurons induced IPSCs in principal neurons of the BLA/BMA (Fig. $5 G$ ). The synaptic delay of the IPSC was significantly longer compared with the monosynaptic EPSC recorded at $-80 \mathrm{mV}$, revealing their disynaptic nature $\left(t_{(11)}=7.01, p<0.001\right.$, paired $t$ test, $n=12$ cells; Fig. $\left.5 G\right)$. The IPSCs were mediated by the inhibitory neurotransmitter GABA because they were inhibited by bicuculline, a $\mathrm{GABA}_{\mathrm{A}}$ receptor antagonist $\left(t_{(5)}=2.74, p<0.05\right.$, paired $t$ test; $n=6$ cells; Fig. $5 H)$. The IPSCs were also blocked by glutamate receptor inhibitors (NBQX + D-AP5), confirming the disynaptic feedforward inhibition $\left(t_{(5)}=4.34, p<0.01\right.$, paired $t$ test; $n=6$ cells; Fig. $\left.5 I\right)$. Together, these results suggest that double-projecting vCA1 neurons make functional synapses onto the basal amygdala, inducing both monosynaptic excitatory and feedforward inhibitory responses.

We also estimated the proportion of presynaptic inputs from double-projecting vCA1 neurons to overall inputs from vCA1 neurons projecting to the basal amygdala. To this end, we injected CAV2-Cre and AAV-DIO-ChR2-eYFP into the basal amygdala and $\mathrm{VH}$, respectively (Fig. 5J). Under these conditions, local blue light illumination applied to the basal amygdala activated ChR2expressing presynaptic axons of vCA1 neurons projecting to the basal amygdala alone (single-projecting neurons), as well as double-projecting vCA1 neurons. In the basal amygdala, EPSCs induced by optogenetic stimulation of both single- and doubleprojecting vCA1 neurons (Fig. $5 K$ ) were larger than EPSCs in-

\footnotetext{
(Figure legend continued.) bicuculine ( $30 \mu \mathrm{m}$, red trace). Inset, Quantification of EPSC inhibition by bicuculline ( ${ }^{*} p<0.05, n=6$ cells). $I$, Representative traces of IPSCs induced and recorded as described in $\boldsymbol{G}$. The black trace is IPSCs recorded under control conditions. IPSCs were completely blocked by the application of NBQX (10 $\mu \mathrm{M})+\mathrm{D}-\mathrm{AP5}(50 \mu \mathrm{M})$ (red trace). Inset, Quantification of EPSC inhibition by NBQX (10 $\mu \mathrm{m})+\mathrm{D}-\mathrm{AP5}$ (** $p<0.01, n=6$ cells).J, Experimental setup for $\boldsymbol{K}$. Retrograde CAV2-Cre was injected into the basal amygdala (BLA/ BMA) and AAV-DIO-ChR2-eYFP was injected into the VH CA1 area, resulting in the expression of Cre recombinase ( $\mathrm{Cre}+)$ and ChR2-eYFP (ChR2 + ) in vCA1 neurons projecting to the basal amygdala. Local blue light illumination onto the amygdala activated the presynaptic axons of vCA1 neurons projecting to the basal amygdala alone (single-projecting neurons), as well as VCA1 neurons projecting to both the MPFC and amygdala (double-projecting neurons), which induced postsynaptic responses in the basal amygdala (Rec). $\boldsymbol{K}$, Representative traces of EPSCS induced by photostimulation of axons of amygdala-projecting vCA1 neurons (single and double projecting). EPSCs were evoked by photostimulation ( $470 \mathrm{~nm}$ blue LED, $20 \mathrm{~mW} / \mathrm{mm}^{2}, 1 \mathrm{~ms}$ duration, blue vertical bar) applied locally to the amygdala and were recorded in a BMA neuron at $-80 \mathrm{mV}$ in voltage-clamp mode, as described in $\boldsymbol{F}$. Note the different vertical scale bars in $\boldsymbol{F}$ and $\boldsymbol{K} . \boldsymbol{L}$, Quantification of the peak amplitudes of EPSCs recorded in the basal amygdala and induced by photostimulation, as described in $\boldsymbol{F}$ and $\boldsymbol{K}$. Photostimulations of the same light intensity and duration ( $20 \mathrm{~mW} / \mathrm{mm}^{2}, 1 \mathrm{~ms}$ duration) were applied to activate the axons of both single- and double-projecting vCA1 neurons, as described in $\boldsymbol{J}$ and $\boldsymbol{K}$, or stimulate presynaptic inputs only from double-projecting vCA1 neurons, as described in $\boldsymbol{A}$ and $\boldsymbol{F}$. EPSCs induced by optogenetic stimulation of both single-and double-projecting vCA1 neurons were significantly larger than EPSCs induced by activation of double-projecting vCA1 neurons alone ${ }^{* * *} p<$ $0.001) . n=23$ cells for single/double-projecting vCA1 axons and $n=24$ cells for doubleprojecting vCA1 axons. Error bars indicate SEM.
}

duced by activation of double-projecting vCA1 neurons alone in previous experiments $\left(t_{(29)}=6.29, p<0.001\right.$, unpaired $t$ test; $n=$ 23 cells for single/double-projecting vCA1 axons and $n=24$ cells for double-projecting vCA1 axons; Fig. $5 L$ ). The average peak amplitude of EPSCs induced by stimulation of double-projecting vCA1 neurons was $23.0 \%$ of the average amplitude of EPSCs evoked by activation of both single- and double-projecting vCA1 neurons, which was close to the proportion of double-projecting neurons to overall vCA1 neurons projecting to the amygdala from our retrograde tracing experiments (16.6\%; Fig. $3 F, G)$.

\section{Double-projecting vCA1 neurons make excitatory synapses onto mPFC neurons}

We next investigated whether double-projecting vCA1 neurons also form functional synapses with mPFC neurons. We induced the expression of ChR2-eYFP selectively in amygdala-projecting CA1 hippocampal neurons by injecting CAV2-Cre into the basal amygdala and AAV-DIO-ChR2-eYFP into the VH (Fig. 6A). The amygdala-projecting $\mathrm{CA} 1$ neurons were identified in the $\mathrm{VH}$ with eYFP fluorescence (Fig. 6B). In the amygdala, we observed eYFPlabeled axons of amygdala-projecting vCA1 neurons (Fig. 6C). eYFP-labeled axons were also detected in the mPFC (Fig. 6D), indicating that these axons originated from vCA1 hippocampal neurons projecting to both the amygdala and $\mathrm{mPFC}$. These axon collaterals were found in both the PL and IL divisions of the $\mathrm{mPFC}$. In the PL, axons of double-projecting vCA1 neurons were found predominantly in layers $2-3$, whereas they were detected in both layers 2-3 and layers 5-6 in the IL (Fig. 6E,F). Local blue light illumination of these ChR2-expressing axons induced EPSCs in $\mathrm{mPFC}$ neurons at $-80 \mathrm{mV}$ in voltage-clamp mode (Fig. $6 G$ ). The peak amplitude of EPSC was proportional to the photostimulation intensity (Fig. 6G,H). The average amplitude of EPSCs was significantly larger in the PL neurons than in IL neurons (Fig. $6 H$; main effect of $\mathrm{mPFC}$ area, IL/PL: $F_{(1,57)}=5.28, p<$ 0.05 ; main effect of photostimulation intensity: $F_{(3,57)}=51.61$, $p<0.001 ; \mathrm{mPFC}$ area $\times$ photostimulation intensity: $F_{(3,57)}=1.33$, $p=0.27$; repeated-measures two-way ANOVA. $n=10-11$ neurons for PL and IL), suggesting that the PL receives more presynaptic inputs from the double-projecting vCA1 neurons than the IL. As in the basal amygdala, optogenetic stimulation of the axon collaterals of double-projecting vCA1 neurons induced IPSCs at $0 \mathrm{mV}$ in the $\mathrm{mPFC}$ neurons with a more delayed onset than the EPSCs, indicating disynaptic feedforward inhibition (Fig. 6I). There was no significant difference in the IPSC amplitude or the EPSC/IPSC ratio between the PL and IL (IPSC: $t_{(13)}=0.07, p=$ 0.95 ; EPSC/IPSC ratio: $t_{(12)}=0.83, p=0.42$; unpaired $t$ test; $n=$ $8-11$ cells for PL and IL; Figure $6 I-L)$. Together, these results suggest that double-projecting vCA1 neurons make excitatory synapses onto $\mathrm{mPFC}$ neurons.

\section{Activation of double-projecting vCA1 neurons induces action potential firings in amygdala-projecting $\mathrm{mPFC}$ neurons}

Our results suggest that double-projecting vCA1 neurons are activated preferentially during context exposure (Fig. 4) and activate both the mPFC and amygdala simultaneously through axon collaterals (Figs. 5, 6). If mPFC neurons activated by these axon collaterals are connected to BLA/BMA neurons through excitatory synapses, then double-projecting vCA1 neurons could activate $\mathrm{VH}-$ mPFC-BLA/BMA pathway. We tested this possibility by investigating whether optogenetic stimulation of double-projecting vCA1 neurons could activate $\mathrm{MPFC}$ neurons projecting to the basal amygdala. To this end, we injected CAV2-Cre into the basal amygdala (BLA/BMA) and AAV-DIO-ChR2-eYFP into the vCA1 

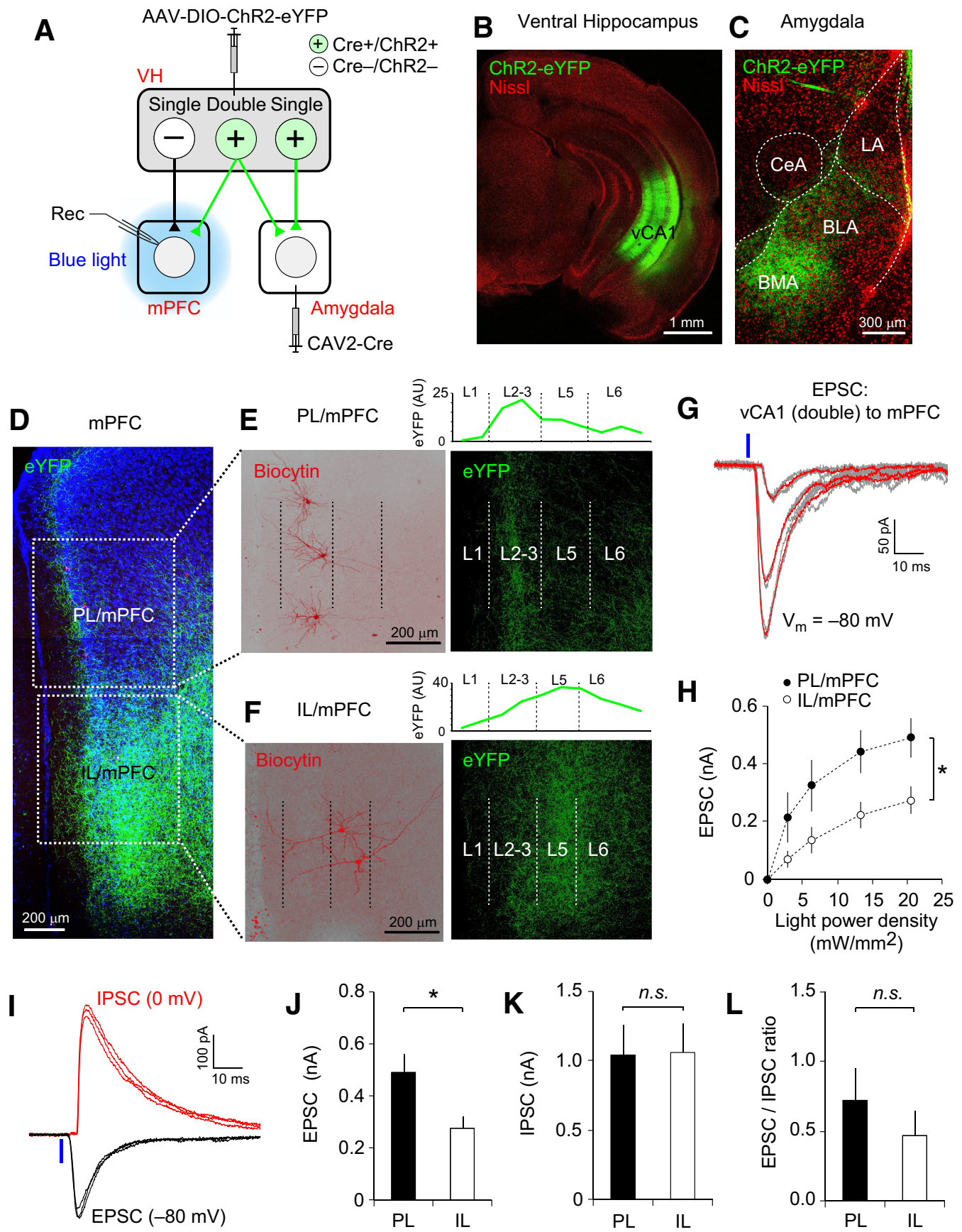

Figure 6. Double-projecting vCA1 neurons form functional synapses onto mPFC neurons. A, Experimental setup. Retrograde CAV2-Cre was injected into the basal amygdala (BLA/BMA) and AAV-DIO-ChR2eYFP was injected into the VH, inducing the expression of Cre recombinase and ChR2-eYFP (Cre +/ChR2 +$)$ in vCA1 neurons projecting to the basal amygdala. Local blue light illumination onto the mPFC activated the ChR2-expressing axons of double-projecting vCA1 neurons, inducing postsynaptic responses in the mPFC (Rec). VCA1 neurons projecting to the mPFC, but not to the amygdala, did not express Cre or ChR2 (Cre-/ChR2 -) and their axons were not activated by local photostimulation. B, C, Confocal microscopic images of the VH (B) and amygdala ( $($ ) taken 4 weeks after viral injection surgery, as described in $\boldsymbol{A} . \boldsymbol{B}$, Green fluorescence in the VH indicates CA1 pyramidal neurons projecting to the basal amygdala. $C$, Green fluorescence in the amygdala indicates axon fibers of amygdala-projecting vCA1 neurons. Red fluorescence indicates Nissl staining. D, Microscopicimage showing eYFP-labeled axons (green) of double-projecting vCA1 neurons in the mPFC. Parts of the PLand IL divisions of the mPFC are marked with square dotted lines and are shown in $\boldsymbol{E}$ and $\boldsymbol{F}$, respectively. Blue fluorescence indicates DAPI staining. $\boldsymbol{E}$, Magnified images of the PL/mPFC in $\boldsymbol{D}$. Left, Three pyramidal neurons in layers $2-3$ of the PL/mPFC were labeled with biocytin/streptavidin-Alexa Fluor 568 (red) after electrophysiological recording. Right, Same area of the PL/mPFC as in the left panel showing the axonal fibers of double-projecting vCA1 neurons (green). These projections were found predominantly in layers $2-3(\mathrm{~L} 2-\mathrm{L} 3)$ of the $\mathrm{PL} / \mathrm{mPFC}$. The borders between the cortical layers are indicated as dotted vertical lines. The graph above the image indicates the relative intensity of eYFP (green, arbitrary unit, AU) in the PL/mPFC. F, Magnified images of the IL/mPFC in D. Left, Two pyramidal neurons in L5 of the IL/mPFC were labeled with biocytin/streptavidin-Alexa Fluor 568 (red) after electrophysiological recording. Right, Same area of the IL/mPFC as in the left panel showing the axonal fibers of double-projecting vCA1 neurons (green). These projections were found in L2-L3 and L5-L6) of the IL/mPFC. The borders between the cortical layers are indicated as dotted vertical lines. The graph above the image indicates the relative intensity of eYFP in the IL/mPFC. G, Representative traces of EPSCS recorded in an $\mathrm{mPFC}$ neuron. EPSCS were evoked by pulses of photostimulation ( $470 \mathrm{~nm}$ blue LED, $1 \mathrm{~ms}$ duration, blue vertical bar) of the ChR2-expressing axons of double-projecting vCA1 neurons and were recorded in the $\mathrm{mPFC}$ neuron at $-80 \mathrm{mV}$ in voltage-clamp mode. Three groups of EPSCs were evoked by photostimulation with different light intensities (2.8, 6.4, and 13.4 $\mathrm{mW} / \mathrm{mm}^{2}$ ). The red traces are the averages of individual EPSCS (gray traces). $\boldsymbol{H}$, Graph showing the average amplitudes of EPSCs induced and recorded as described in $\boldsymbol{G}$, which are plotted versus photostimulation intensity. The peak amplitudes of EPSCs in the PL/mPFC (filled circles) were significantly larger than in the IL/mPFC (open circles). ${ }^{*} p<0.05 . n=10$ and 11 neurons ( 4 mice) for PL and IL, respectively. I, Representative traces of EPSCs (black traces) and IPSCs (red traces) recorded in an mPFC neuron. The EPSCs and IPSCs were induced as described in $\mathbf{G}$ and recorded at -80 and $0 \mathrm{mV}$, respectively, in voltage-clamp mode in the same mPFC neuron. Note a more delayed onset of IPSC than EPSC after photostimulation (20 mW/mm², blue vertical line).(Figure legend continues.) 

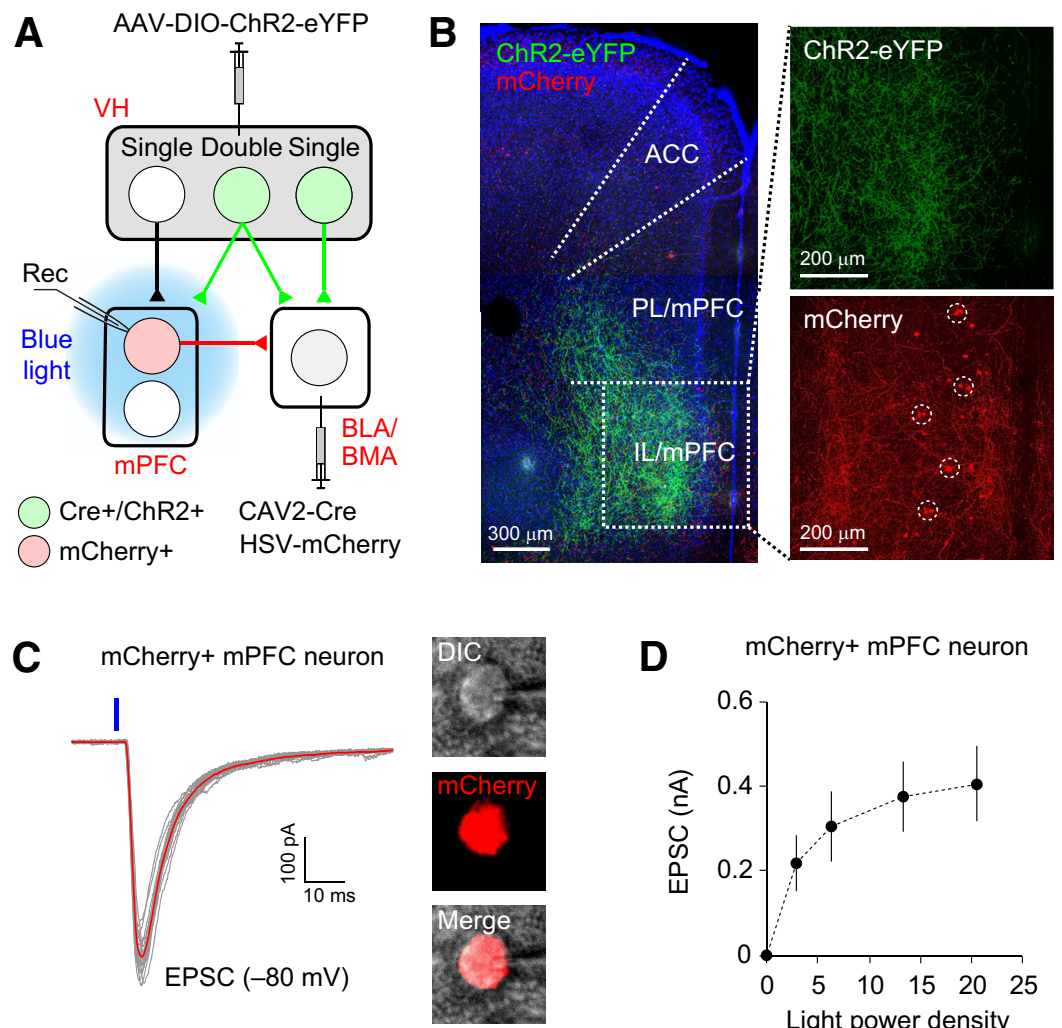

E AP: mCherry+ mPFC neuron

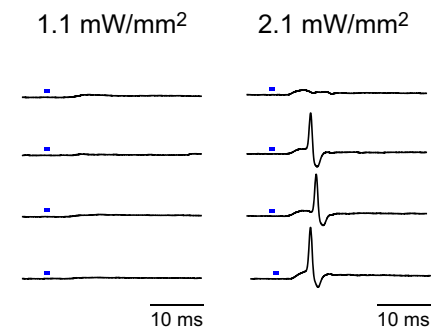

$2.8 \mathrm{~mW} / \mathrm{mm}^{2}$
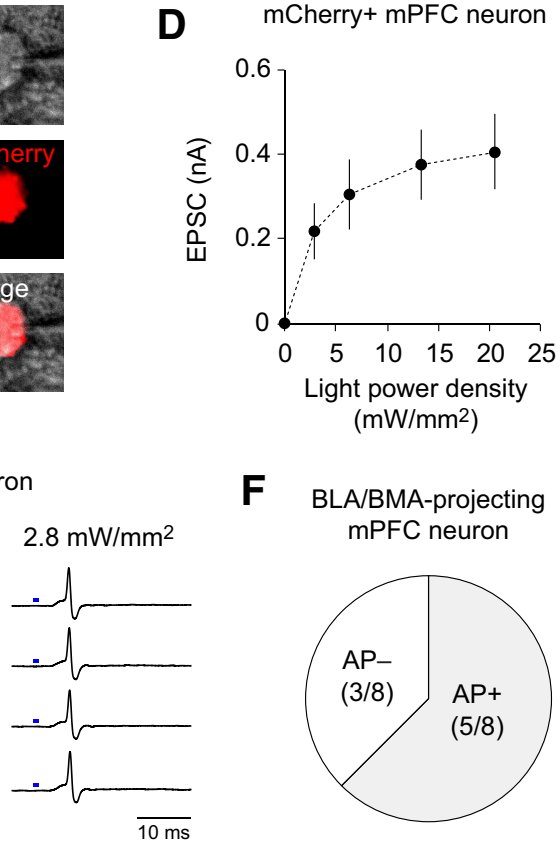

$\mathbf{F}$

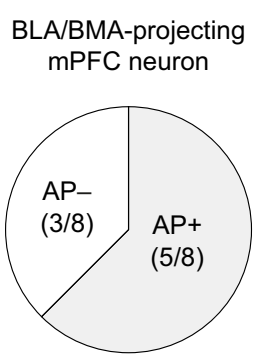

Figure 7. Double-projecting vCA1 neurons activate amygdala-projecting mPFC neurons of the VH-mPFC-amygdala circuit. $\boldsymbol{A}$, Experimental setup. Retrograde CAV2-Cre was injected into the basal amygdala (BLA/BMA) and AAV-DI0-ChR2-eYFP was injected into the VH to induce ChR2-eYFP expression in the vCA1 neurons projecting to the basal amygdala. Another retrograde virus, HSV-mCherry, was also injected into the basal amygdala to label the BLA/BMA-projecting mPFC neurons with mCherry. Local blue light illumination onto the mPFC activated the axons of double-projecting CA1 neurons expressing ChR2-eYFP, which induced the postsynaptic responses recorded in BLA/BMA-projecting mPFC neurons (Rec). $\boldsymbol{B}$, Left, Microscopic image showing the eYFPlabeled axons of double-projecting vCA1 neurons in the MPFC (green). Blue fluorescence represents DAPI staining. A portion of the $\mathrm{IL} / \mathrm{mPFC}$ (a dotted square) is magnified in the right panels. Top right, eYFP-labeled axons of double-projecting vCA1 neurons in the IL/mPFC (green). Bottom right, mCherry-labeled IL/mPFC neurons projecting to the basal amygdala (red, dotted circles). ACC, Anterior cingulate cortex. $C$, Left, Representative traces of EPSCs recorded in an amygdala-projecting mPFC neuron. EPSCs were induced by photostimulation ( $470 \mathrm{~nm}$ blue LED, 1 ms duration, blue vertical bar) of the ChR2-expressing axons of doubleprojecting vCA1 neurons and recorded in mCherry-labeled $\mathrm{mPFC}$ neurons at $-80 \mathrm{mV}$ in voltage-clamp mode. The red trace is the average of the individual EPSCs (gray traces). Right, Microscopic images of the mCherry-labeled recorded neuron confirming that the $\mathrm{mPFC}$ neuron projects to the basal amygdala. DIC, Differential interference contrast microscopic image. D, Graph showing the average peak amplitudes of EPSCs recorded, as described in $C$, which are plotted versus photostimulation intensity. $n=10$ neurons from 2 mice. Error bars indicate SEM. $\boldsymbol{E}$, Representative traces of action potential (AP) firings recorded in an mCherry-labeled mPFC neuron in cell-attached mode. The intensity of photostimulation (blue bars) is indicated above each column. $\boldsymbol{F}$, Photostimulation of double-projecting vCA1 neurons induced AP firings in 5 of 8 amygdala-projecting mPFC neurons examined (AP $+: 62.5 \%)$.

\section{$\leftarrow$}

(Figure legend continued.) $\quad \boldsymbol{J}-\boldsymbol{L}$, Quantification of the peak amplitudes of the EPSC $(\boldsymbol{H})$ and IPSC $(\boldsymbol{K})$ and the EPSC/IPSC ratio $(\boldsymbol{L})$ in postsynaptic PL/mPFC and IL/mPFC neurons. In each $\mathrm{mPFC}$ neuron, both the EPSCs and IPSCS were evoked by photostimulation of the same intensity (20 $\mathrm{mW} / \mathrm{mm}^{2}$ ). The average EPSC was significantly larger in the PL/mPFC than in the IL/mPFC (J, $\left.{ }^{*} p<0.05\right)$. There was no significant difference in IPSC amplitude $(\boldsymbol{K} ;$ n.s., not significant $)$ or the EPSC/IPSC ratio ( $L$ ) between the PL and IL (n.s., not significant). $n=8-10$ cells ( 4 mice) for the PL and $n=8-11$ cells ( 4 mice) for the IL. Error bars indicate SEM.
$\left(\mathrm{mW} / \mathrm{mm}^{2}\right)$

area to induce ChR2-eYFP expression in BLA/BMA-projecting vCA1 neurons (Fig. $7 A)$. We also injected the retrograde tracer HSV-mCherry into the basal amygdala to label BLA/BMA-projecting mPFC neurons with mCherry. In brain slices of the mPFC, BLA/BMA-projecting mPFC neurons were identified with mCherry, and the axon collaterals of double-projecting vCA1 neurons were identified with eYFP (Fig. $7 B$ ). Local blue light illumination of the $\mathrm{mPFC}$ induced EPSCs in BLA/BMA-projecting $\mathrm{mPFC}$ neurons $(n=10$ cells; Fig. $7 C, D)$. Moreover, optogenetic stimulation of the projections of double-projecting vCA1 neurons induced action potential firings in 5 of 8 BLA/BMA-projecting $\mathrm{mPFC}$ neurons examined (Fig. $7 E, F$ ), indicating that double-projecting vCA1 neurons can activate BLA/BMA-projecting mPFC neurons. Because the mPFC projects to the basal amygdala (BLA/BMA) with glutamatergic excitatory synapses (Cho et al., 2013), our results suggest that double-projecting vCA1 neurons can activate the VH-mPFCamygdala pathway. By activating both the direct $\mathrm{VH}$-amygdala pathway and the indirect $\mathrm{VH}-\mathrm{mPFC}$-amygdala pathway, doubleprojecting vCA1 neurons could induce more robust excitatory synaptic responses in the basal amygdala through temporal summation than vCA1 neurons projecting only to the amygdala. These synaptic mechanisms could enable doubleprojecting vCA1 neurons to convey contextual information more efficiently to the basal amygdala for the expression of contextual fear memory (see model in Fig. 8).

\section{Discussion}

Our results demonstrate the synaptic mechanisms of how double-projecting vCA1 neurons modulate the activity of the $\mathrm{mPFC}$ and basal amygdala. Our viral tracing and electrophysiological studies revealed that the $\mathrm{VH}$ projects directly to the $\mathrm{mPFC}$ and amygdala with excitatory synapses. Optogenetic silencing of the vCA1 inhibited the recall of contextual fear memory in mice, suggesting that $\mathrm{VH}$ activity is required for contextual fear responses. In retrograde tracing experiments, a proportion of vCA1 neurons projected to both the mPFC and amygdala. Moreover, compared with the vCA1 neurons projecting to either the mPFC or amygdala alone, a greater proportion of double-projecting vCA1 neurons were recruited during context exposure, indicating their role in encoding context representations. At the synaptic level, optogenetic stimulation of double-projecting vCA1 neurons induced monosynaptic excitatory and feedforward inhibitory responses in the mPFC and amygdala, indicating 
that they could convey contextual information to these brain areas simultaneously through the $\mathrm{VH}-\mathrm{mPFC}$ and $\mathrm{VH}-$ amygdala pathways. Furthermore, the activation of double-projecting vCA1 neurons was sufficient to induce AP firings in $\mathrm{mPFC}$ neurons projecting to the basal amygdala, suggesting their role in activating the VH-mPFC-amygdala circuit. With these synaptic mechanisms, double-projecting vCA1 neurons could induce synchronized neural activity in the mPFC and amygdala and convey contextual information efficiently to the basal amygdala through both the direct $\mathrm{VH}-$ amygdala pathway and the indirect VH-mPFC-amygdala pathway. By modulating the activity of the mPFC and basal amygdala, double-projecting vCA1 neurons are likely to contribute to the acquisition and retrieval of contextual fear memory (Fig. 8B).

Previous studies have suggested that a proportion of vCA1 neurons project to both the mPFC and amygdala (Ishikawa and Nakamura, 2006; Jin and Maren, 2015). Consistently, electrical and optogenetic stimulation of the mPFC and amygdala has been shown to evoke antidromic responses in the same vCA1 neurons (Ishikawa and Nakamura, 2006; Ciocchi et al., 2015). However, the antidromic responses may also have been induced by activation of the axon fibers of passage and do not represent faithfully the functional synaptic connections. Moreover, the extent of collateralization of vCA 1 neural populations projecting to the $\mathrm{mPFC}$ and amygdala was not estimated in these studies. Using dual retrograde viral tracing, we found that $18.0 \pm 1.5 \%$ of $\mathrm{mPFC}$-projecting vCA1 neurons also projected to the amygdala and that $16.6 \pm 1.2 \%$ of amygdala-projecting vCA1 neurons also projected to the $\mathrm{mPFC}$. With electrophysiological recordings, we also demonstrated that optogenetic stimulation of the axon collaterals of double-projecting vCA1 neurons induced monosynaptic excitatory and feedforward inhibitory responses in the $\mathrm{mPFC}$ and amygdala, indicating the presence of functional excitatory synaptic connections from double-projecting vCA1 neurons to the mPFC and amygdala.

Prior studies have indicated that pharmacological inhibition and electrolytic lesion of the VH suppress contextual fear expression (Trivedi and Coover, 2004; Pentkowski et al., 2006; Zhang et al., 2015). However, such manipulations of hippocampal function could affect other neuronal processes nonspecifically and do not allow for cellular and temporal precision. To achieve acute and reversible silencing of $\mathrm{VH}$ activity with a high degree of temporal precision, we used an in vivo optogenetic approach and found that contextual fear expression was suppressed by optogenetic silencing of the $\mathrm{VH}$, which suggests a role for the $\mathrm{VH}$ in the retrieval of contextual fear memory, although silencing of $\mathrm{VH}$ activity may have also affected the ability of the subjects to time the expected shock (Balsam et al., 2010; Eichenbaum, 2014). This finding is consistent with a recent study implicating the VHbasal amygdala pathway in contextual fear expression (Xu et al., 2016). Moreover, c-Fos immunohistochemistry re- vealed that double-projecting vCA1 neurons were activated to a greater extent during context exposure and the recall of contextual fear memory than vCA1 neurons projecting to either the mPFC or amygdala alone, suggesting that they play a role in context encoding during contextual fear responses. How, then, do double-projecting vCA1 neurons contribute to the retrieval of contextual fear memory? During the recall of contextual fear memory, the hippocampal representation of a context is conveyed to the MPFC and amygdala, which are essential areas for contextual fear responses (Helmstetter and Bellgowan, 1994; Muller et al., 1997; Corcoran and Quirk, 2007; Goshen et al., 2011; Zhu et al., 2014; Adhikari et al., 2015). Double-projecting vCA1 neurons encoding contextual information can activate the mPFC and amygdala simultaneously through their axon collaterals and induce synchronized neural activity in these areas. Because synchronized activity between the $\mathrm{MPFC}$ and amygdala has been implicated in conditioned fear responses (Lesting et al., 2011), double-projecting vCA1 neurons could contribute to the recall of contextual fear memory by facilitating synchronized activity between these areas. Moreover, our results also indicate that activation of double-projecting vCA1 neurons induced larger excitatory postsynaptic responses in the $\mathrm{PL} / \mathrm{mPFC}$ than in the IL/mPFC. Previous studies have suggested that the $\mathrm{PL} / \mathrm{mPFC}$ and IL/mPFC may play different roles in the modulation of contextual fear responses because the expression of contextual fear memory was suppressed by pharmacological inhibition of the $\mathrm{PL} / \mathrm{mPFC}$, as well as optogenetic activation of the IL/mPFC projecting to the amygdala (Corcoran and Quirk, 2007; Adhikari et al., 2015). Therefore, doubleprojecting vCA1 neurons could facilitate contextual fear responses by activating the $\mathrm{PL} / \mathrm{mPFC}$ more robustly than the $\mathrm{IL} / \mathrm{mPFC}$. 
Previous studies with electrolytic lesions and pharmacological and chemogenetic inhibition have suggested that the $\mathrm{VH}$ is also required for the acquisition and consolidation of contextual fear memory (Maren and Fanselow, 1995; Bast et al., 2001; Zhang et al., 2001; Rudy and Matus-Amat, 2005; Zhu et al., 2014). Doubleprojecting vCA1 neurons could also contribute to these processes by conveying contextual information efficiently to the amygdala for the context-US association. The acquisition of contextual fear memory requires coordinated neural activity in the hippocampus and amygdala (Ohkawa et al., 2015) and the memory of the context-US association is thought to be encoded in the pathway from the hippocampus to the amygdala (Maren and Fanselow, 1995). Contextual representations in the $\mathrm{DH}$ could be conveyed to the amygdala indirectly though the entorhinal and perirhinal cortices for the formation of contextual fear memory (Tanaka et al., 2014). Alternatively, vCA1 hippocampal neurons can also convey contextual information directly to the amygdala through their excitatory

projections for contextual fear memory formation. The results of our electrophysiological study suggest that double-projecting vCA1 neurons can activate both the direct $\mathrm{VH}$-amygdala pathway and the indirect $\mathrm{VH}-\mathrm{mPFC}$-amygdala pathway. By activating direct and indirect pathways to the amygdala, they can induce successive postsynaptic responses in the basal amygdala with short time intervals, resulting in efficient temporal summation (Fig. 8B). The coactivation of convergent inputs with short time intervals induces input timing-dependent synaptic plasticity (ITDP) in the amygdala, a type of long-term potentiation that has been implicated in the formation of conditioned fear memory (Cho et al., 2011). Therefore, double-projecting vCA1 neurons conveying contextual information could facilitate ITDP induction in the basal amygdala and contribute to the encoding of context-US associative memory in the $\mathrm{VH}$ to the amygdala pathway.

One of the limitations of our dual retrograde tracing study is that only the mPFC and amygdala were examined as projection targets of the VH. Therefore, labeled vCA1 neurons were classified as neurons projecting to either the $\mathrm{MPFC}$ or amygdala alone or neurons projecting to both the $\mathrm{mPFC}$ and amygdala. These vCA1 neurons may also have long-range projections to other brain areas including the nucleus accumbens (NAc). We observed that a population of $\mathrm{mPFC}$ - or amygdala-projecting $\mathrm{VH}$ neurons also have axon collaterals projecting to the NAc (J.-H.C., unpublished data). Therefore, vCA1 neurons identified in our study could have been further assigned into subgroups if the NAc had also been included in their projection target area and vCA1 neurons of each subgroup may play different roles in behavioral tasks and emotional states. In support of this, a recent study has suggested that vCA1 neurons with triple projections to the mPFC, amygdala, and NAc show more prominent firing during behavioral tasks and sharp wave/ripples than vCA1 neurons with single or double projections to these areas (Ciocchi et al., 2015). Moreover, vCA1 neurons projecting to the NAc have been implicated in goal-directed behavior and social memory in rodents (Ciocchi et al., 2015; Okuyama et al., 2016).

In addition to the $\mathrm{VH}$, long-range axon collaterals were also found in other brain areas. For example, principal neurons in the BLA project to multiple downstream targets including the NAc, $\mathrm{CeA}$, and $\mathrm{VH}$ with $17-33 \%$ collateralization in mice (Beyeler et al., 2016). Therefore, long-range axon collaterals to multiple brain areas may be a general feature of the neural circuits in the brain and could promote synchronized neural activity and longterm synaptic plasticity in different brain areas. The experimental approaches used in our current study enable the selective stimulation of axon collaterals and detailed electrophysiological analyses of synaptic responses, which can be used to determine synaptic connections of other brain areas projecting to multiple targets with long-range axon collaterals. Our results also have implications for optogenetic and chemogenetic approaches to control the activity of a neural circuit, in which opsins or designer receptors are expressed in a population of neurons projecting to a specific brain area. Therefore, long-range collateralization to multiple brain areas should be considered when designing experiments using these approaches and interpreting the experimental results.

\section{References}

Adhikari A, Lerner TN, Finkelstein J, Pak S, Jennings JH, Davidson TJ, Ferenczi E, Gunaydin LA, Mirzabekov JJ, Ye L, Kim SY, Lei A, Deisseroth K (2015) Basomedial amygdala mediates top-down control of anxiety and fear. Nature 527:179-185. CrossRef Medline

Atasoy D, Aponte Y, Su HH, Sternson SM (2008) A FLEX switch targets Channelrhodopsin-2 to multiple cell types for imaging and long-range circuit mapping. J Neurosci 28:7025-7030. CrossRef Medline

Balsam PD, Drew MR, Gallistel CR (2010) Time and associative learning. Comp Cogn Behav Rev 5:1-22. CrossRef Medline

Bast T, Zhang WN, Feldon J (2001) The ventral hippocampus and fear conditioning in rats. Different anterograde amnesias of fear after tetrodotoxin inactivation and infusion of the GABA(A) agonist muscimol. Exp Brain Res 139:39-52. CrossRef Medline

Beyeler A, Namburi P, Glober GF, Simonnet C, Calhoon GG, Conyers GF, Luck R, Wildes CP, Tye KM (2016) Divergent routing of positive and negative information from the amygdala during memory retrieval. Neuron 90:348-361. CrossRef Medline

Boyden ES, Zhang F, Bamberg E, Nagel G, Deisseroth K (2005) Millisecondtimescale, genetically targeted optical control of neural activity. Nat Neurosci 8:1263-1268. CrossRef Medline

Cho JH, Bayazitov IT, Meloni EG, Myers KM, Carlezon WA Jr, Zakharenko SS, Bolshakov VY (2011) Coactivation of thalamic and cortical pathways induces input timing-dependent plasticity in amygdala. Nat Neurosci 15:113-122. CrossRef Medline

Cho JH, Deisseroth K, Bolshakov VY (2013) Synaptic encoding of fear extinction in mPFC-amygdala circuits. Neuron 80:1491-1507. CrossRef Medline

Chow BY, Han X, Dobry AS, Qian X, Chuong AS, Li M, Henninger MA, Belfort GM, Lin Y, Monahan PE, Boyden ES (2010) High-performance genetically targetable optical neural silencing by light-driven proton pumps. Nature 463:98-102. CrossRef Medline

Ciocchi S, Passecker J, Malagon-Vina H, Mikus N, Klausberger T (2015) Brain computation: selective information routing by ventral hippocampal CA1 projection neurons. Science 348:560-563. CrossRef Medline

Corcoran KA, Quirk GJ (2007) Activity in prelimbic cortex is necessary for the expression of learned, but not innate, fears. J Neurosci 27:840-844. CrossRef Medline

Denny CA, Kheirbek MA, Alba EL, Tanaka KF, Brachman RA, Laughman KB, Tomm NK, Turi GF, Losonczy A, Hen R (2014) Hippocampal memory traces are differentially modulated by experience, time, and adult neurogenesis. Neuron 83:189-201. CrossRef Medline

Eichenbaum H (2014) Time cells in the hippocampus: a new dimension for mapping memories. Nat Rev Neurosci 15:732-744. CrossRef Medline

Franklin KBJ and Paxinos G (2008) The Mouse Brain in Stereotaxic Coordinates. San Diego, CA: Academic Press.

Goshen I, Brodsky M, Prakash R, Wallace J, Gradinaru V, Ramakrishnan C, Deisseroth K (2011) Dynamics of retrieval strategies for remote memories. Cell 147:678-689. CrossRef Medline

Helmstetter FJ, Bellgowan PS (1994) Effects of muscimol applied to the basolateral amygdala on acquisition and expression of contextual fear conditioning in rats. Behav Neurosci 108:1005-1009. CrossRef Medline

Hnasko TS, Perez FA, Scouras AD, Stoll EA, Gale SD, Luquet S, Phillips PE, Kremer EJ, Palmiter RD (2006) Cre recombinase-mediated restoration of nigrostriatal dopamine in dopamine-deficient mice reverses hypophagia and bradykinesia. Proc Natl Acad Sci U S A 103:8858-8863. CrossRef Medline

Hübner C, Bosch D, Gall A, Lüthi A, Ehrlich I (2014) Ex vivo dissection of 
optogenetically activated mPFC and hippocampal inputs to neurons in the basolateral amygdala: implications for fear and emotional memory. Front Behav Neurosci 8:64. CrossRef Medline

Ishikawa A, Nakamura S (2006) Ventral hippocampal neurons project axons simultaneously to the medial prefrontal cortex and amygdala in the rat. J Neurophysiol 96:2134-2138. CrossRef Medline

Jay TM, Witter MP (1991) Distribution of hippocampal CA1 and subicular efferents in the prefrontal cortex of the rat studied by means of anterograde transport of Phaseolus vulgaris leucoagglutinin. J Comp Neurol 313:574-586. CrossRef Medline

Jin J, Maren S (2015) Fear renewal preferentially activates ventral hippocampal neurons projecting to both amygdala and prefrontal cortex in rats. Sci Rep 5:8388. CrossRef Medline

Jung MW, Wiener SI, McNaughton BL (1994) Comparison of spatial firing characteristics of units in dorsal and ventral hippocampus of the rat. J Neurosci 14:7347-7356. Medline

Kishi T, Tsumori T, Yokota S, Yasui Y (2006) Topographical projection from the hippocampal formation to the amygdala: a combined anterograde and retrograde tracing study in the rat. J Comp Neurol 496:349368. CrossRef Medline

Kjelstrup KB, Solstad T, Brun VH, Hafting T, Leutgeb S, Witter MP, Moser EI, Moser MB (2008) Finite scale of spatial representation in the hippocampus. Science 321:140-143. CrossRef Medline

Lesting J, Narayanan RT, Kluge C, Sangha S, Seidenbecher T, Pape HC (2011) Patterns of coupled theta activity in amygdala-hippocampalprefrontal cortical circuits during fear extinction. PLoS One 6:e21714. CrossRef Medline

Liu X, Ramirez S, Pang PT, Puryear CB, Govindarajan A, Deisseroth K, Tonegawa S (2012) Optogenetic stimulation of a hippocampal engram activates fear memory recall. Nature 484:381-385. CrossRef Medline

Maren S, Fanselow MS (1995) Synaptic plasticity in the basolateral amygdala induced by hippocampal formation stimulation in vivo. J Neurosci 15:7548-7564. Medline

Maren S, Phan KL, Liberzon I (2013) The contextual brain: implications for fear conditioning, extinction and psychopathology. Nat Rev Neurosci 14:417-428. CrossRef Medline

Mattis J, Tye KM, Ferenczi EA, Ramakrishnan C, O'Shea DJ, Prakash R, Gunaydin LA, Hyun M, Fenno LE, Gradinaru V, Yizhar O, Deisseroth K (2011) Principles for applying optogenetic tools derived from direct comparative analysis of microbial opsins. Nat Methods 9:159-172. CrossRef Medline

Muller J, Corodimas KP, Fridel Z, LeDoux JE (1997) Functional inactivation of the lateral and basal nuclei of the amygdala by muscimol infusion prevents fear conditioning to an explicit conditioned stimulus and to contextual stimuli. Behav Neurosci 111:683-691. CrossRef Medline

Ohkawa N, Saitoh Y, Suzuki A, Tsujimura S, Murayama E, Kosugi S, Nishi- zono H, Matsuo M, Takahashi Y, Nagase M, Sugimura YK, Watabe AM, Kato F, Inokuchi K (2015) Artificial association of pre-stored information to generate a qualitatively new memory. Cell Rep 11:261-269. CrossRef Medline

Okuyama T, Kitamura T, Roy DS, Itohara S, Tonegawa S (2016) Ventral CA1 neurons store social memory. Science 353:1536-1541. CrossRef Medline

Pentkowski NS, Blanchard DC, Lever C, Litvin Y, Blanchard RJ (2006) Effects of lesions to the dorsal and ventral hippocampus on defensive behaviors in rats. Eur J Neurosci 23:2185-2196. CrossRef Medline

Pitkänen A, Pikkarainen M, Nurminen N, Ylinen A (2000) Reciprocal connections between the amygdala and the hippocampal formation, perirhinal cortex, and postrhinal cortex in rat: a review. Ann N Y Acad Sci 911: 369-391. Medline

Rudy JW, Matus-Amat P (2005) The ventral hippocampus supports a memory representation of context and contextual fear conditioning: implications for a unitary function of the hippocampus. Behav Neurosci 119:154-163. CrossRef Medline

Strange BA, Witter MP, Lein ES, Moser EI (2014) Functional organization of the hippocampal longitudinal axis. Nat Rev Neurosci 15:655-669. CrossRef Medline

Tanaka KZ, Pevzner A, Hamidi AB, Nakazawa Y, Graham J, Wiltgen BJ (2014) Cortical representations are reinstated by the hippocampus during memory retrieval. Neuron 84:347-354. CrossRef Medline

Trivedi MA, Coover GD (2004) Lesions of the ventral hippocampus, but not the dorsal hippocampus, impair conditioned fear expression and inhibitory avoidance on the elevated T-maze. Neurobiol Learn Mem 81:172184. CrossRef Medline

Xu C, Krabbe S, Gründemann J, Botta P, Fadok JP, Osakada F, Saur D, Grewe BF, Schnitzer MJ, Callaway EM, Lüthi A (2016) Distinct hippocampal pathways mediate dissociable roles of context in memory retrieval. Cell 167:961-972.e16. CrossRef Medline

Zhang CL, Houbaert X, Lepleux M, Deshors M, Normand E, Gambino F, Herzog E, Humeau Y (2015) The hippocampo-amygdala control of contextual fear expression is affected in a model of intellectual disability. Brain Struct Funct 220:3673-3682. CrossRef Medline

Zhang WN, Bast T, Feldon J (2001) The ventral hippocampus and fear conditioning in rats: different anterograde amnesias of fear after infusion of N-methyl-D-aspartate or its noncompetitive antagonist MK801 into the ventral hippocampus. Behav Brain Res 126:159-174. CrossRef Medline

Zhu H, Pleil KE, Urban DJ, Moy SS, Kash TL, Roth BL (2014) Chemogenetic inactivation of ventral hippocampal glutamatergic neurons disrupts consolidation of contextual fear memory. Neuropsychopharmacology 39:1880-1892. CrossRef Medline 